\title{
U-Pb zircon in situ dating with LA-MC-ICP-MS using a mixed detector configuration
}

\author{
FARID CHEMALE Jr ${ }^{1}$, KOJI KAWASHITA ${ }^{2}$, IVO A. DUSSIN ${ }^{2}$, JANAÍNA N. ÁVILA ${ }^{3}$, \\ DAYVISSON JUSTINO ${ }^{4}$ and ANELISE BERTOTTI ${ }^{4}$ \\ ${ }^{1}$ Instituto de Geociências, Universidade de Brasília, Campus Darcy Ribeiro, 70910-900 Brasília, DF, Brasil \\ ${ }^{2}$ Centro de Pesquisas Geocronológicas, Universidade de São Paulo, Rua do Lago, 562, 05508-080 São Paulo, SP, Brasil \\ ${ }^{3}$ Research School of Earth Sciences, Australian National University, Building 47, Daley Road, Canberra, ACT 0200, Australia \\ ${ }^{4}$ Curso de Pós-Graduação em Geociências, Universidade Federal do Rio Grande do Sul, \\ Av. Bento Gonçalves, 9500, Caixa Postal 15001, 91501-970 Porto Alegre, RS, Brasil
}

Manuscript received on September 1, 2010; accepted for publication on April 4, 2011

\begin{abstract}
The LA-MC-ICP-MS method applied to U-Pb in situ dating is still rapidly evolving due to improvements in both lasers and ICP-MS. To test the validity and reproducibility of the method, 5 different zircon samples, including the standard Temora-2, ranging in age between $2.2 \mathrm{Ga}$ and $246 \mathrm{Ma}$, were dated using both LAMC-ICP-MS and SHRIMP. The selected zircons were dated by SHRIMP and, after gentle polishing, the laser spot was driven to the same site or on the same zircon phase with a $213 \mathrm{~nm}$ laser microprobe coupled to a multi-collector mixed system. The data were collected with a routine spot size of $25 \mu \mathrm{m}$ and, in some cases, of 15 and $40 \mu \mathrm{m}$. A careful cross-calibration using a diluted U-Th-Pb solution to calculate the Faraday reading to counting rate conversion factors and the highly suitable GJ-1 standard zircon for external calibrations were of paramount importance for obtaining reliable results. All age results were concordant within the experimental errors. The assigned age errors using the LA-MC-ICP-MS technique were, in most cases, higher than those obtained by SHRIMP, but if we are not faced with a high resolution stratigraphy, the laser technique has certain advantages.
\end{abstract}

Key words: MC-ICP-MS, SHRIMP, Faraday cups, Multi-ion Counting System, U-Pb in situ zircon dating.

\section{INTRODUCTION}

Accurate $\mathrm{U}-\mathrm{Pb}$ ages have been obtained from zircons by isotope dilution thermal ionization mass spectrometry (ID-TIMS) (as described by, e.g., Bowring et al.1998, Mundil et al. 2001, 2004). The advent of in situ analyses at the microscale by laser ablation (LA)-ICP-MS and ion microprobe (e.g., SHRIMP) has shown the complexity of zircons grains, which often exhibit more than one crystallization

Correspondence to: Farid Chemale Junior

E-mail: fchemale@unb.br phase associated with different geological processes. Of these methods, ID-TIMS presents more precise and accurate data when a single phase zircon is dated (i.e., associated with one main geological event). However, ion microprobe analyses (such as those provided by SHRIMP) have better spatial resolution for dating the different growth phases on single zircon grains, often producing $\mathrm{U}-\mathrm{Pb}$ data as precise as the ID-TIMS method.

LA-ICP-MS is well accepted as a reliable and more convenient method of dating detrital zircons (provenance information applied to sedimentary basin 
evolution), especially after the introduction of the 213 $\mathrm{nm}$ laser wavelength. This method allows the analysis of spots at the size conventionally used during the SHRIMP analysis, which is the best method for in situ age determinations. During the last decade, numerous studies were published presenting successful U-Pb zircon data using ICP-MS (e.g., Koesler and Sylvester 2003, Jackson et al. 2004). The development of MC-ICP-MS configured with Faradays cup and multiplier ion counting channels has introduced the possibility of generating $\mathrm{U}-\mathrm{Pb}$ zircon data that may be comparable to those using SHRIMP (Cocherie and Robert 2008). For the present work, the amounts of ${ }^{238} \mathrm{U},{ }^{232} \mathrm{Th}$ and ${ }^{206} \mathrm{~Pb}$ were obtained with Faraday cups, while ${ }^{202} \mathrm{Hg},{ }^{204} \mathrm{~Pb},{ }^{204} \mathrm{Hg}$ and ${ }^{207} \mathrm{~Pb}$ were obtained with MIC (multiplier ion counting) channels because these isotopes occur in very low amounts in most zircons. To test the suitability of our system and the U-Pb age reproducibility of the Concordia ages, zircon samples covering a wide age range from 2.2 to $0.2 \mathrm{Ga}$ with a large variation of ${ }^{206} \mathrm{~Pb} /{ }^{238} \mathrm{U}$, ${ }^{232} \mathrm{Th} /{ }^{238} \mathrm{U}$ and ${ }^{207} \mathrm{~Pb} /{ }^{206} \mathrm{~Pb}$ ratios were analyzed by both SHRIMP and LA-MC-ICPMS. The analyzed samples comprised the following: i) zircons from the early Paleozoic gabbrodiorite (Temora II sample provided by the Australian Geological Survey); ii) zircons from Paleoproterozoic Tandilla gneisses (provided by Orestes Santos); iii) Neoproterozoic Piquiri Syenite; and iv) zircon samples of the volcanic rock of the Rincon Blanco basin.

\section{ANALYTICAL PRocedures}

Zircons were separated by conventional procedures using heavy liquids and magnetic separator after the concentration by hand panning. The most clear and inclusion-free zircons from the least magnetic fractions were hand picked for U-Pb SHRIMP and LA-MC-ICP-MS analyses.

SHRIMP

U-Pb SHRIMP zircon geochronology was carried out at the Research School of Earth Sciences,
Australian National University, and at the Department of Geology and Geophysics, University of Western Australia by using SHRIMP II and RG equipments. Handpicked zircons were mounted in epoxy discs along with zircon standards, ground and polished, microphotographed in transmitted and reflected light, and and their internal zoning imaged by cathodoluminescence (CL) using scanning electron microscope. The mounts were then cleaned and gold-coated in preparation for the SHRIMP analysis. Analytical methods and data treatment can be found elsewhere (Compston et al. 1984, Williams 1998). Zircons grains were analyzed with a $2-3 \mathrm{nA}, 10 \mathrm{kV}$ primary $\mathrm{O}_{2}$ - beam focused to a $\sim 25$ to $\sim 20 \mu \mathrm{m}$ diameter spot. At mass resolution 5500 the $\mathrm{Pb}$, Th and $\mathrm{U}$ isotopes were resolved from all major interferences. The reduction of raw data and age calculation were carried out using Squid 2.02 and Isoplot-Ex (Ludwig 2003). U and Th concentrations were determined relative to those measured in the RSES standard SL13

\section{LA-MC-ICP-MS}

The same samples used for SHRIMP analyses were dated by LA-MC-ICP-MS with the New Wave UP213 Laser Ablation Microprobe coupled to a MC-ICP-MS Neptune at the Isotope Geology Laboratory of the Rio Grande do Sul University. We have tried to do the spot with the same size or somewhat larger and at the same site or close as regarding those did with SHRIMP. In some cases, due to the low content of $\mathrm{Pb}$, either larger spots were used or increased the number of spots (in the same zircon phase) in order to get more precise data with the LAM method (see discussion of results). Isotope data were acquired in static mode with spot sizes of 25 and $40 \mu \mathrm{m}$. Laser-induced elemental fractional and instrumental mass discrimination were corrected using the reference zircon (GJ-1) 
(Jackson et al. 2004). Two GJ-1 analyses were measured after every four or ten sample zircon spots. The external error was calculated after the propagation error of the GJ-1 mean and the individual sample zircon (or spot).

\section{Collector configuration}

The collector configuration used for simultaneous measurements of $\mathrm{Th}, \mathrm{U}, \mathrm{Pb}$ and $\mathrm{Hg}$ isotopes is as follows:

\begin{tabular}{|l|c|c|c|c|c|c|c|c|}
\hline Position & & & L4 & & L3 & Axial & H2 & H4 \\
\hline Faraday cup & $202 \mathrm{Hg}$ & $\begin{array}{c}204 \mathrm{Hg}+ \\
204 \mathrm{~Pb}\end{array}$ & $206 \mathrm{~Pb}$ & $207 \mathrm{~Pb}$ & $208 \mathrm{~Pb}$ & & 232Th & $238 \mathrm{U}$ \\
\hline $\begin{array}{l}\text { Multiplier Ion } \\
\text { Counting Channel }\end{array}$ & MIC3 & MIC4 & & MIC6 & & & & \\
\hline
\end{tabular}

The gain calibration of Faraday cups and the cross calibration between the L4 cup against the MIC3, 4 and 6 were carried out before the laser running was started. The MIC3 to 4 are attached to the L4 Faraday cup, and the MIC6 is attached to the L3.

Because the multicollector system involves 3 ion counters and 5 conventional Faraday collectors, the gain and cross calibration must be performed routinely. The gain factor used to calibrate the Faraday measurements is calculated by applying a constant signal of 33.0 volts. The cross calibration used to calculate the necessary conversion factors (voltage to cps) is achieved using a $220 \mathrm{ppt}$ Neptune solution with the addition of 200 ppt Th and an efficient nebulizer system. A calculated conversion value of $62,500 \mathrm{cps} / \mathrm{mV}$ was used.

The various ratios are obtained simultaneously and appropriately corrected. However, because of inherent elemental and isotopic fractionations during laser ablation, these ratios vary during the analysis and require different approaches to estimate reliable data. As illustrated for the standard zircon, the ${ }^{207} \mathrm{~Pb} /{ }^{206} \mathrm{~Pb}$ ratios do not fractionate visibly like the ${ }^{206} \mathrm{~Pb} /{ }^{238} \mathrm{U}$ ratios, which involve two different elements with their own chemical and physical properties (Fig. 1). $\mathrm{Pb}$ is more volatile than $U$, which condenses progressively on the walls of the pit formed during the laser ablation process. We routinely adopt the average of the ${ }^{207} \mathrm{~Pb} /{ }^{206} \mathrm{~Pb}$ determinations as the representative value for the sample, and for ${ }^{206} \mathrm{~Pb} /{ }^{238} \mathrm{U}$, we assume the extrapolated value for $\mathrm{t}$ (time) $=0$. Outliers that do not show a good alignment are also discarded. Other ratios, such as ${ }^{206} \mathrm{~Pb} /{ }^{207} \mathrm{~Pb}$ and ${ }^{232} \mathrm{Th} /{ }^{238} \mathrm{U}$, are also taken into account in the extrapolated ratios when they are applicable or exhibit the same trend of fractionation. These ratios are usually quite close to the expected values.

The conversion factors are calculated based on the available data for the standard used and applied to unknown samples. Thus, a homogeneous standard is of paramount importance. The GJ-1 standard (GEMOC ARC National Key Center) meets the requirements for the methods used in our laboratory, and the ratios of ${ }^{206} \mathrm{~Pb}^{*} / 238 \mathrm{U}$, ${ }^{207} \mathrm{~Pb}^{*} /{ }^{206} \mathrm{~Pb}^{*}$ and ${ }^{232} \mathrm{Th} /{ }^{238} \mathrm{U}$ are homogeneous during the entire "bracket" technique, a standardsamples-standard analysis.

\section{Mass bias correction, external correction, and laser conditions}

The isotope ratios and inter-element fractionation of data obtained by the MC-ICP-MS instrument were evaluated by interspersing the GJ-1 standard zircon on every set of $4,6,8$ or 10 zircon samples (spots). The number of analyzed spots varied 
depending on the zircon homogeneity and the amount of $\mathrm{Pb}$ and $\mathrm{U}$ in the zircon. The GJ-1 standard zircon was used to estimate the necessary corrections for the external corrections and the internal instrumental fractionation. The GJ-1 zircon and sample were assembled in the same mounting. The spot size of the laser was usually $25 \mu \mathrm{m}$, but the spot sizes were $40 \mu \mathrm{m}$ and $15 \mu \mathrm{m}$ for the zircon phases with a low amount of ${ }^{207} \mathrm{~Pb}$ (under 10,000 cps) and for small zircon grains $(<30 \mu \mathrm{m}$ of diameter), respectively.

The repetition rate of the laser was $10 \mathrm{~Hz}$. The energy varied from 0.3 to $1.1 \mathrm{~mJ} /$ pulse, and the corresponding spot sizes varied from $15 \mu \mathrm{m}$ to $40 \mu \mathrm{m}$. The data acquisition occurred in 50 cycles of $1.048 \mathrm{~s}$ of integration time, and the masses 202, 204, 206, 207, 208, 232, and 238 were collected simultaneously. For every standard and sample set, blank values in the same conditions as the standard and sample were also measured. The average blank values were subtracted from all individual cycle measurements. The ${ }^{204} \mathrm{~Pb}$ value was corrected for ${ }^{204} \mathrm{Hg}$ by assuming a ${ }^{202} \mathrm{Hg} /{ }^{204} \mathrm{Hg}$ ratio equal to 4.355 . Laser operation conditions are shown in Table I.

\section{Common Pb correction}

The usual method for common-lead corrections on zircon grains (based on the non-radiogenic $204 \mathrm{~Pb}$ isotope) is not appropriate when using the laser technique because the ${ }^{204} \mathrm{~Pb}$ signal is strongly affected by ${ }^{204} \mathrm{Hg}$. The majority of the ${ }^{204} \mathrm{Hg}$ comes from gases ( $\mathrm{Ar}$ and $\mathrm{He}$ ) that are required in the ICP and ablation procedures. After the $\mathrm{Hg}$ correction based on ${ }^{202} \mathrm{Hg}$ is measured, the common ${ }^{204} \mathrm{~Pb}$ is insignificant in most situations. For instance, a typical signal intensity of the ${ }^{204} \mathrm{Hg}$ during laser ablation of the standard zircon is in the 600-1000 cps range, and the calculated count rate for $204 \mathrm{~Pb}$ is less than the statistical error of ca. 25-33 cps. We assume that the ${ }^{204} \mathrm{~Pb}$ values obtained from zircon grains contain some common $\mathrm{Pb}$, and we also assume a concordant age of ${ }^{206} \mathrm{~Pb} /{ }^{238} \mathrm{~Pb}$ and ${ }^{207} \mathrm{~Pb} /{ }^{206} \mathrm{~Pb}$ as the estimated age. In this case, we estimate the radiogenic composition of ${ }^{206} \mathrm{~Pb}$ and ${ }^{207} \mathrm{~Pb}$ using the following equation for a fraction of the non-radiogenic ${ }^{206} \mathrm{~Pb}$ (Williams 1998):

$\mathrm{f}_{206}=\left[{ }^{206} \mathrm{~Pb} /{ }^{204} \mathrm{~Pb}\right] \mathrm{c} /\left[{ }^{206} \mathrm{~Pb} /{ }^{204} \mathrm{~Pb}\right]_{\mathrm{s}}$ $\mathrm{f}_{207}=\left[{ }^{207} \mathrm{~Pb} /{ }^{204} \mathrm{~Pb}\right] \mathrm{c} /\left[{ }^{207} \mathrm{~Pb} /{ }^{204} \mathrm{~Pb}\right]_{\mathrm{s}}$

TABLE I

MC-ICP-MS and Laser operating conditions.

\begin{tabular}{|c|c|}
\hline Laser operating conditions & \\
\hline \multirow{2}{*}{ Laser type New Wave } & MC-ICP-MS Neptune \\
Wavelenght $213 \mathrm{~nm}$ & Foward power $1200 \mathrm{w}$ \\
output energy 0.3 to 1.1 & Reflected power $<5 \mathrm{w}$ \\
shot repetition rate $10 \mathrm{hz}$ & Cup configuration: \\
spot size single spot analysis & Faradays ${ }^{206} \mathrm{~Pb},{ }^{208} \mathrm{~Pb},{ }^{232} \mathrm{Th},{ }^{238} \mathrm{U}$ \\
$15,25,40 \mu \mathrm{m}$ & MIC's ${ }^{202} \mathrm{Hg},{ }^{204} \mathrm{Hg}+{ }^{204} \mathrm{~Pb},{ }^{20} 7 \mathrm{~Pb}$ \\
& Gas input: \\
& Coolant flow (Ar) $151 / \mathrm{min}$ \\
& Auxiliary flow $(\mathrm{Ar}) 0.81 / \mathrm{min}$ \\
& Carrier flow $0.751 / \mathrm{min}(\mathrm{Ar})+0.451 / \mathrm{min}(\mathrm{He})$ \\
& Acquisition $50 \mathrm{cycles}$ of $1.048 \mathrm{~s}$ \\
& \\
\hline
\end{tabular}


For the common lead isotope composition, we assume the isotope compositions evolve as proposed by Stacey and Kramers (1975). This assumption is required to determine an initial estimated age.

The ${ }^{207} \mathrm{~Pb} /{ }^{206} \mathrm{~Pb}$ and ${ }^{206} \mathrm{~Pb} /{ }^{238} \mathrm{U}$ ratios were corrected after the $\mathrm{f}_{206}$ and $\mathrm{f}_{207}$ were determined for each cycle. The cycles with values of $f_{206}$ above 0.0025 are not usually included in the age calculation.

\section{Calculation of the ratios and error estimation}

After the blank and common $\mathrm{Pb}$ corrections, the ratios and their absolute errors (one sigma level) of ${ }^{206} \mathrm{~Pb} * / 238 \mathrm{U},{ }^{232} \mathrm{Th} /{ }^{238} \mathrm{U}$, and ${ }^{206} \mathrm{~Pb} * / 207 \mathrm{~Pb} *$ were calculated using an Excel sheet. Because the ${ }^{206} \mathrm{~Pb}^{*} / 238 \mathrm{U}$ usually produces a linear fractionation, we used the intercept method for laser-induced $\mathrm{Pb} / \mathrm{U}$ fractionation to correct the ratio according to the formulation proposed by Youden (1951) and adopted by Koesler et al. (2002). The uncertainty of the fractionation-corrected ratio was calculated as one SD (standard deviation) of the intercept $(\sigma \mathrm{R}(\mathrm{o}))$, which is the isotope ratio at the start of laser ablation. The internal derived errors were calculated in the conventional way by taking account of the uncertainties (1 SD) of the respective background signals (see Fig. 1).

For the ${ }^{232} \mathrm{Th} /{ }^{238} \mathrm{U}$ and ${ }^{207} \mathrm{~Pb} * 206 \mathrm{~Pb} *$ ratios, the mean values were used after discarding the outliers.
In some cases, the ${ }^{232} \mathrm{Th} /{ }^{238} \mathrm{U}$ and ${ }^{207} \mathrm{~Pb} * 206 \mathrm{~Pb} *$ ratios show a slight fractionation. Laser-induced fractionation was applied to obtain the $\mathrm{R}(\mathrm{o})$ of these ratios.

\section{RESULTS}

After reduction, the raw U-Pb data and the calculated ${ }^{206} \mathrm{~Pb}^{*} /{ }^{238} \mathrm{U},{ }^{232} \mathrm{Th} /{ }^{238} \mathrm{U}$ and ${ }^{206} \mathrm{~Pb}^{*} /{ }^{207} \mathrm{~Pb}^{*}$ ratios, along with the U-Pb Concordia diagrams, were obtained using Isoplot version EX (Ludwig 2003). We used the Tera-Wasserburg Concordia diagram (Tera and Wasserburg 1972) for the Phanerozoic to late Neoproterozoic rocks and the normal Concordia diagram for Precambrian samples. A summary of the results is presented in Table II.

\section{PALEOPROTEROZOIC TANDILLHA BASEMENT}

Two samples were dated where the spots were of the same size and at the same site in both LA-MC-ICPMS and SHRIMP (Figures 2 and 3, Tables III and IV). The SHRIMP data have already been presented in Hartmann et al. (2002). The obtained ages for sample TA-01, a tonalitic gneiss outcropping in the Tandilla region, are $2,239 \pm 18 \mathrm{Ma}$ (SHRIMP) and 2,243 $\pm 20 \mathrm{Ma}$ (LA-MC-ICP-MS). The younger syenogranite, which cuts the tonalitic gneiss (TA-01), presents ages of 2,114 $\pm 12 \mathrm{Ma}$ (SHRIMP) and 2,112+38/$28 \mathrm{Ma}$ (LA-MC-ICPMS). In both samples, the

TABLE II

Summary of U-Pb zircon age (Ma) of the analyzed samples. * Forced at origin ( $0 \pm 50 \mathrm{Ma})$,

** Age and error after Black et al. (2004).

\begin{tabular}{|cccccccc|}
\hline Sample & Lithotype & LAM & $\pm(2 \mathrm{SD})$ & SHRIMP & $\pm(2 \mathrm{SD})$ & ID-TIMS & $\pm(2 \mathrm{SD})$ \\
\hline TA-01 & Tonalitic gneiss & $2243^{*}$ & 20 & 2239 & 18 & & \\
TA-10 & Syenogranite & 2112 & $38 /-28$ & 2114 & 12 & & \\
RS-2 & Syenite & 592 & 4.7 & 594 & 4.9 & & 0.22 \\
Temora II & Gabbroic diorite & 418.6 & 4.3 & N/A & & $416.5^{* *}$ & \\
RB-06 & Rhyolite & 244.1 & 3.5 & 246.4 & 1.1 & & \\
\hline
\end{tabular}


a)

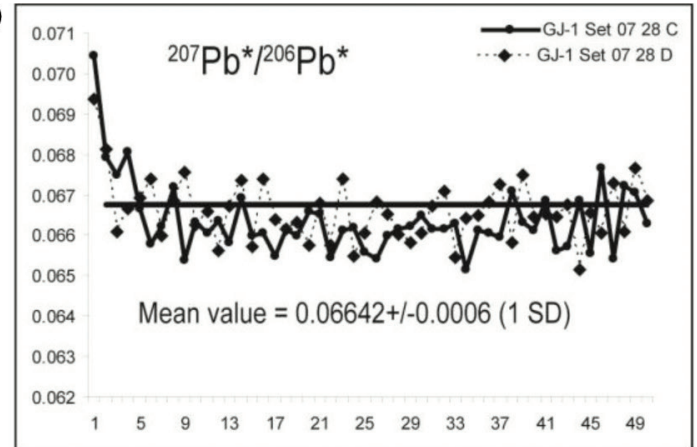

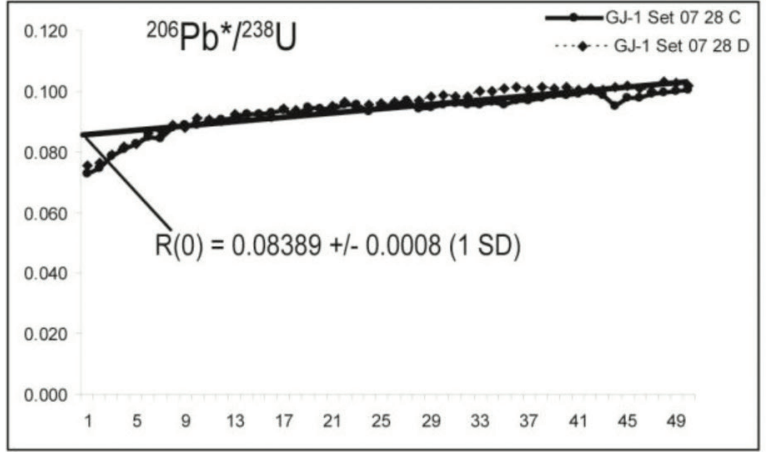

b)

\begin{tabular}{|c|cc|cc|cc|cc|}
\hline & ${ }^{207} \mathrm{~Pb} / /^{206} \mathrm{~Pb}$ & $\mathrm{StDv}$ & ${ }^{206} \mathrm{~Pb} / /^{238} \mathrm{U}$ & $\mathrm{StDv}$ & ${ }^{232} \mathrm{Th} / /^{238} \mathrm{U}$ & $\mathrm{StDv}$ & ${ }^{208} \mathrm{~Pb}{ }^{206} \mathrm{~Pb}$ & \\
ID-TIMS value & $\mathbf{0 . 0 6 0 1 4}$ & & $\mathbf{0 . 0 9 7 5 0}$ & & $\mathbf{0 . 0 2 0 0 0}$ & & $\mathbf{0 . 0 0 6 3}$ & \\
& & & & & & & & \\
GJ-1 Set 28 c & 0.06628 & 0.00066 & 0.08371 & 0.0009 & 0.01908 & 0.0007 & 0.0068 & 0.0028 \\
$\begin{array}{c}\text { GJ-1 Set 28 d } \\
\text { Mean }\end{array}$ & 0.06655 & 0.00060 & 0.08406 & 0.0008 & 0.01864 & 0.0003 & 0.0065 & 0.0023 \\
& $\mathbf{0 . 0 6 6 4 2}$ & & $\mathbf{0 . 0 8 3 8 9}$ & & $\mathbf{0 . 0 1 8 8 6}$ & & $\mathbf{0 . 0 0 6 7}$ & \\
Conversion factor & $\mathbf{0 . 9 0 5 4 5}$ & $\mathbf{0 . 0 0 0 6 3}$ & $\mathbf{1 . 1 6 2 2 9}$ & $\mathbf{0 . 0 0 0 8}$ & $\mathbf{1 . 0 6 0 6 0}$ & $\mathbf{0 . 0 0 0 5}$ & $\mathbf{0 . 9 3 9 8}$ & $\mathbf{0 . 0 0 2 6}$ \\
\hline
\end{tabular}

c)
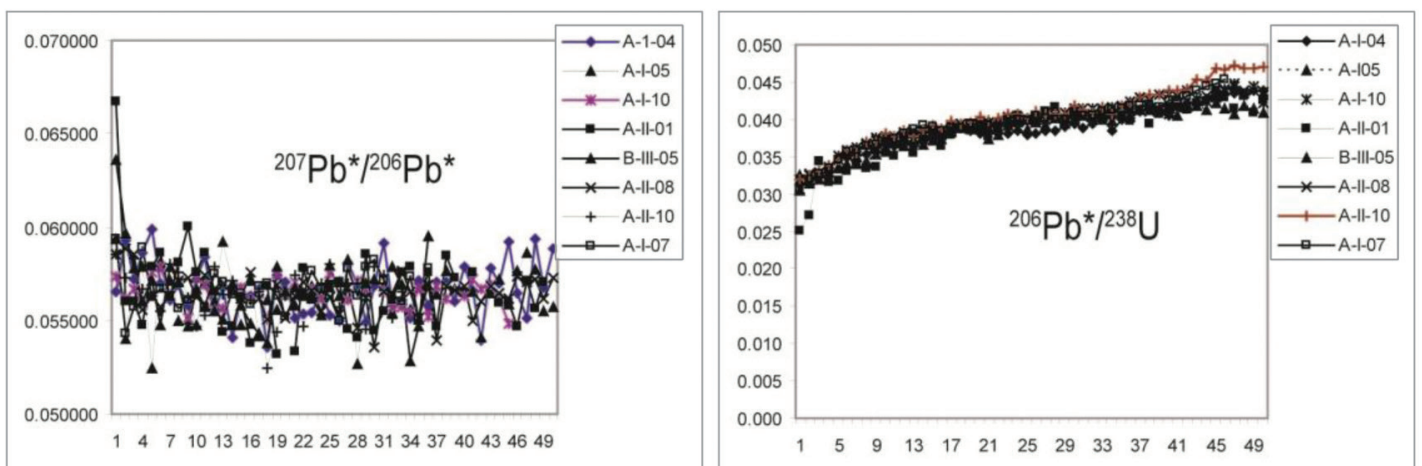

d)

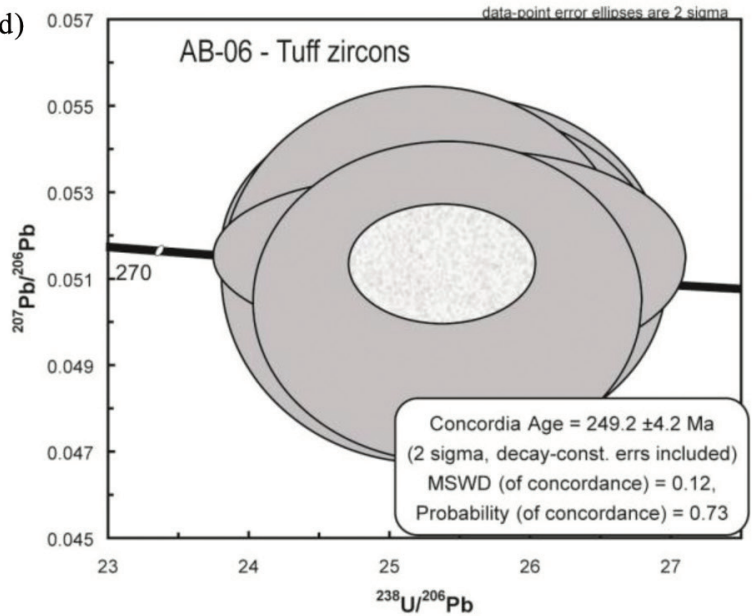

Figure 1. Set of two standard measurements and 8 zircon spots of the AB-06 tuff a) Plot of ${ }^{207} \mathrm{~Pb}^{*} /{ }^{206} \mathrm{~Pb}^{*}$ and ${ }^{206} \mathrm{~Pb}^{*}{ }^{238} \mathrm{U}$ ratios obtained on GJ-1 standard zircon; b) Summary of calculated results on standards; c) Plot of 207Pb*/206Pb* and 206 $\mathrm{Pb} * / 238 \mathrm{U}$ ratios obtained on 8 zircons (AB-06 tuff zircons) in the same analytical conditions as compared to those for GJ-1 standard zircon; d) Concordia age of the analyzed zircons on Tera-Wasserburg diagram. 
results have similar accuracy and precision, but the individual analyses obtained by SHRIMP have lower uncertainties (Fig. 4). The youngest rocks presented two zircons with greater errors, which were attributed to the unstable analytical conditions of the laser during the analyses. Figure 5 shows the results of the GJ-1 standard and zircon spot TA-1 (4-1) before the application of the conversion factor and error propagation.
NEOPROTEROZOIC PIQUIRI SYENITE

The Piquiri Syenite, located in the Sul-RioGrandense Shield in southernmost Brazil, has two zircon phases. The youngest zircon phase, interpreted to be the igneous crystallization of the Piquiri Syenite, was dated by LA-MC-ICPMS at $592 \pm 2.3 \mathrm{Ma}$ and by SHRIMP at $594 \pm 2.7$ (Fig. 6 and Table V). Although both ages are in the
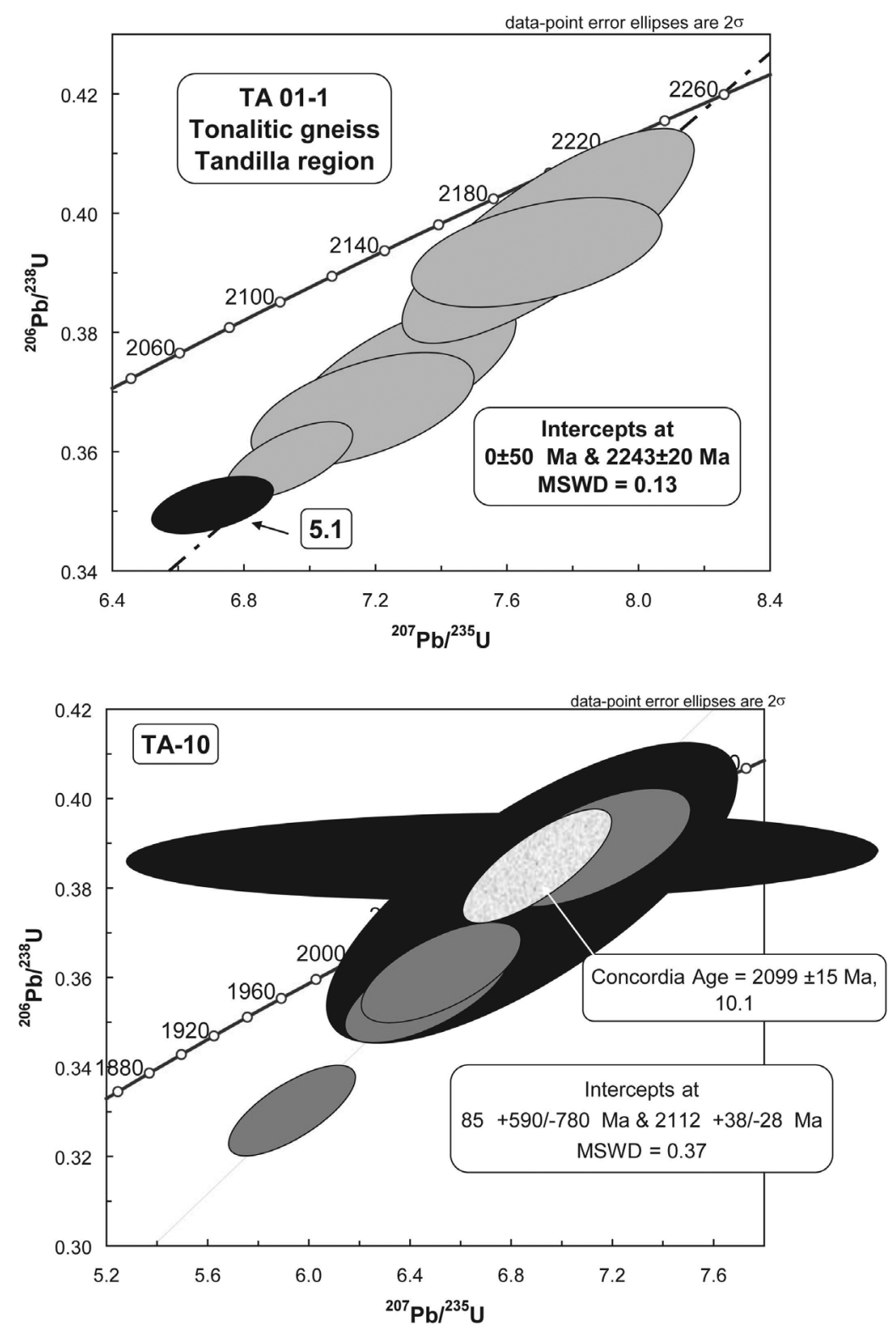

Figure 2. Concordia diagrams with LA-MC-ICP-MS results from samples of the Tandilla region (Argentina). 

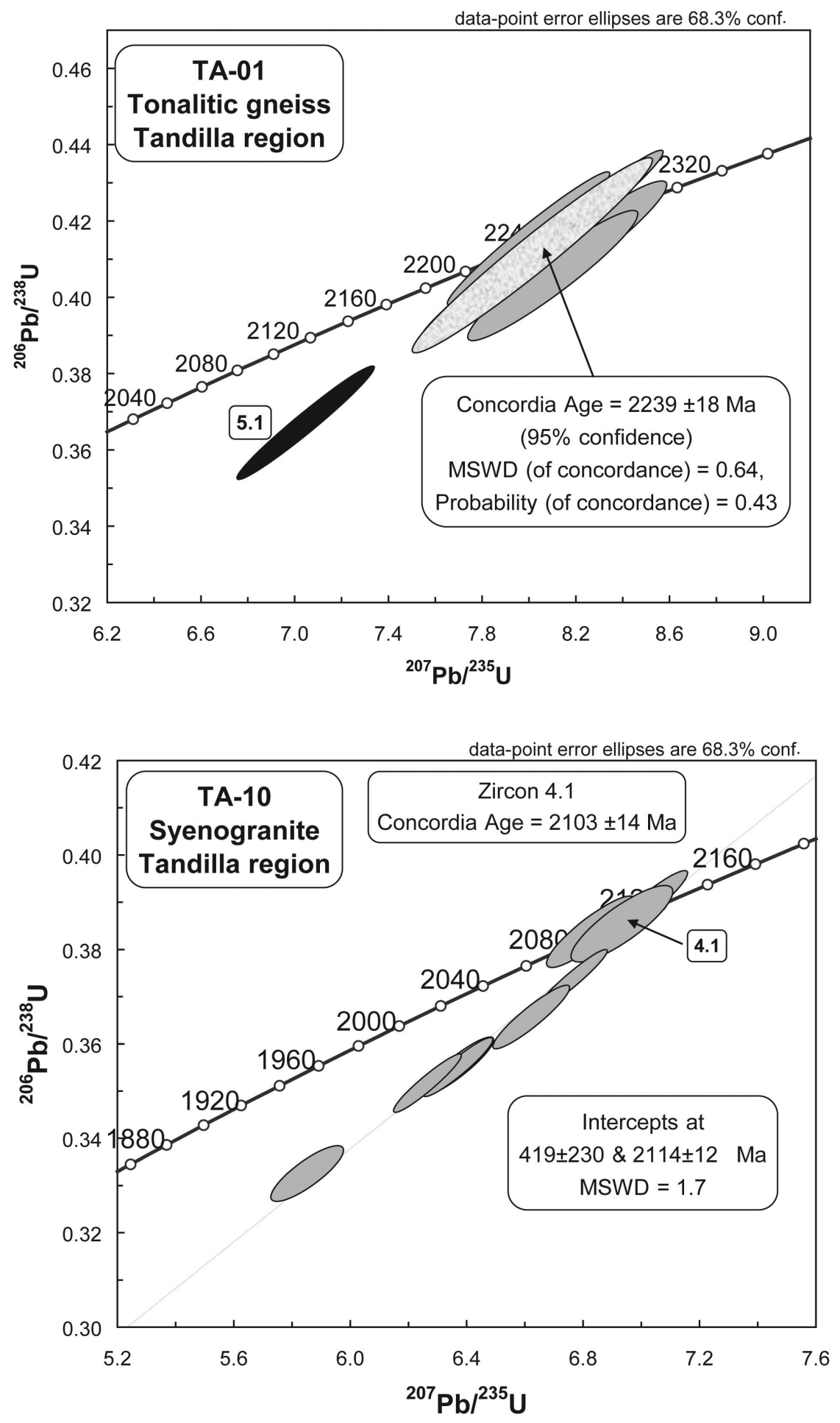

Figure 3. Concordia diagrams with SHRIMP results from samples of the Tandilla region (Argentina). 

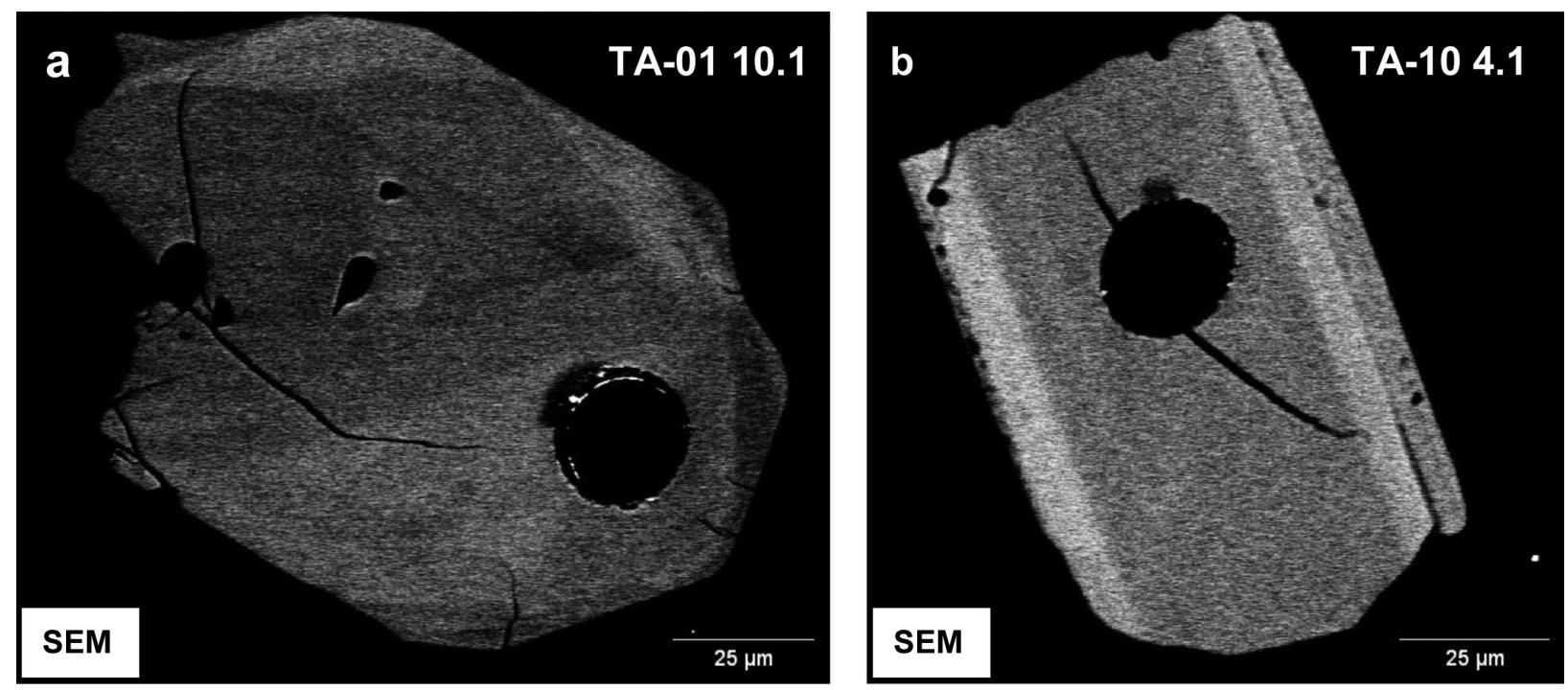

\section{$2247 \pm 48 /-45 \mathrm{Ma}$ LA-MC-ICP-MS}

\section{$2100 \pm 42 \mathrm{Ma}$}

LA-MC-ICP-MS

\section{$2247 \pm 11 \mathrm{Ma}$}

SHRIMP

\section{$2103 \pm 14 \mathrm{Ma}$}

SHRIMP

Figure 4. a) Spot 10.1 of TA-01 sample with results of the SHRIMP and Laser (spot at same site and with the same size $=25 \mu \mathrm{m}$ ) b) Spot 4.1 of TA-10 sample with results of the SHRIMP and Laser (spot at the same site and with the same size $=25 \mu \mathrm{m}$ ).

\section{Standard GJ-1}

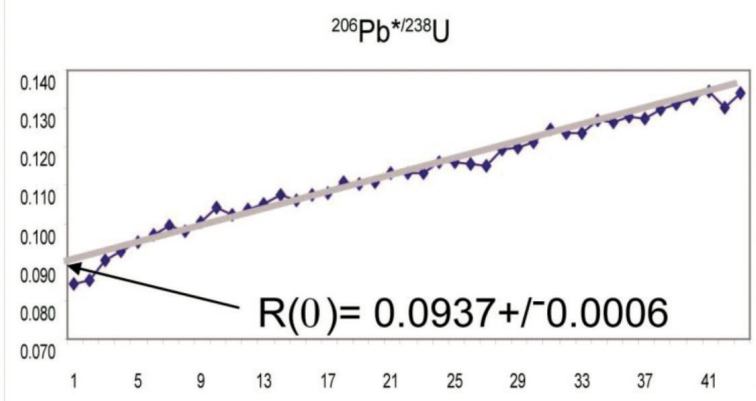

${ }^{207} \mathrm{~Pb}^{* / 206} \mathrm{~Pb}^{*}$

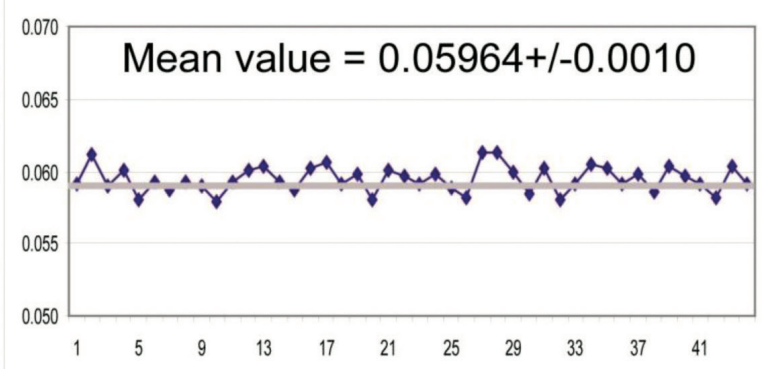

Spot TA 1 (4-1)
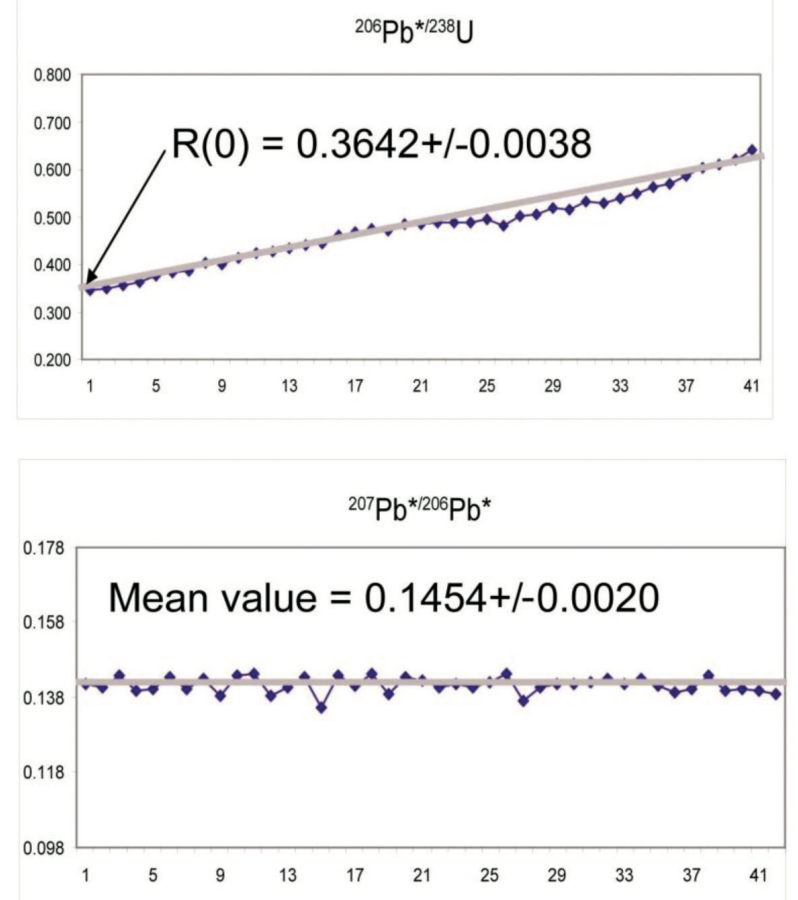

Figure 5. Plot diagram with ${ }^{206} \mathrm{~Pb} * /^{238} \mathrm{U}$ and ${ }^{207} \mathrm{~Pb} *{ }^{206} \mathrm{~Pb} *$ ratios of Spot TA-01 4.1 obtained with LA-MC-ICP-MS $25 \mu \mathrm{m}$ spot size. 


\begin{tabular}{|c|c|c|c|c|c|c|}
\hline ơ & $a$ & 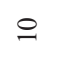 & $\simeq$ & $\nabla$ & in & $\simeq$ \\
\hline H & $\bar{m}$ & ల & तี & लె & q & 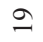 \\
\hline 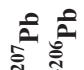 & 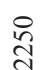 & $\stackrel{\infty}{\stackrel{d}{d}}$ & $\stackrel{ }{\stackrel{\Xi}{(}}$ & 竎 & $\vec{a}$ & $\stackrel{\sim}{\tilde{a}}$ \\
\hline H & テ & $F$ & $\approx$ & $\approx$ & $\Varangle$ & $\stackrel{d}{d}$ \\
\hline : & g & 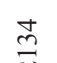 & $\hat{\varrho}$ & $\overrightarrow{\tilde{N}}$ & $\stackrel{\infty}{\circ}$ & 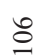 \\
\hline H & $\stackrel{\sim}{\infty}$ & $\tilde{A}$ & $=$ & q & $\bar{\lambda}$ & $\simeq$ \\
\hline D. & 荢 & $\stackrel{\vec{\sim}}{\overrightarrow{1}}$ & $\stackrel{\infty}{\approx}$ & $\stackrel{\tilde{n}}{\sim}$ & $\underset{\vec{N}}{\vec{J}}$ & 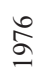 \\
\hline$\Xi \bar{z}$ & $\begin{array}{l}R \\
0\end{array}$ & $\hat{n}$ & oे & $\hat{\imath}$ & 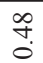 & 0 \\
\hline$\alpha^{\circ} \bar{c}$ & $\stackrel{m}{m}$ & $\underline{\text { o }}$ & $\stackrel{n}{n}$ & $\stackrel{\circ}{\infty}$ & $\stackrel{\circ}{\circ}$ & $\stackrel{t}{i}$ \\
\hline 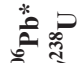 & $\stackrel{m}{m}$ & हn & $\vec{n}$ & ફે & dे & ले \\
\hline$\therefore$ & $n$ & $\sigma$ & $\underline{n}$ & $n$ & $\delta$ & \pm \\
\hline & & & 0 & d & $\infty$ & \\
\hline 突 & 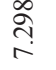 & $\stackrel{2}{\leftrightarrows}$ & 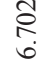 & $\stackrel{\frac{7}{2}}{i}$ & $\stackrel{\infty}{\stackrel{\infty}{ }}$ & $\hat{n}$ \\
\hline$\partial^{\circ} \vdots$ & ले & $\vec{\sigma}$ & $\stackrel{\infty}{\circ}$ & $\stackrel{q}{g}$ & $\stackrel{\infty}{\stackrel{2}{ }}$ & $\stackrel{\infty}{\infty}$ \\
\hline 葛 & $\stackrel{\partial}{\vec{\Xi}}$ & $\bar{\Xi}$ & 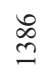 & $\stackrel{O}{\exists}$ & $\stackrel{\partial}{\vec{\Xi}}$ & \& \\
\hline$\odot \vdots$ & $n$ & s & $ஜ$ & $\infty$ & ஓ & I \\
\hline$D^{*}$ & & & & & & \\
\hline 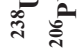 & $\stackrel{\stackrel{b}{i}}{i}$ & $\stackrel{i}{i}$ & $\stackrel{\infty}{i}$ & $\ddot{n}$ & $\stackrel{\vec{n}}{i}$ & $\stackrel{i}{i}$ \\
\hline 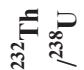 & $\stackrel{n}{0}$ & $\stackrel{\infty}{n}$ & $\stackrel{7}{\div}$ & 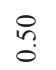 & $\hat{n}$ & กิ \\
\hline ્ֻરે & $\overrightarrow{\bar{\sigma}}$ & 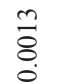 & $\begin{array}{l}\text { } \\
\vdots \\
0\end{array}$ & $\begin{array}{l}n \\
\stackrel{a}{0} \\
0\end{array}$ & 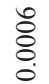 & $\overline{8}$ \\
\hline 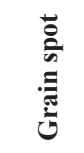 & $\begin{array}{l}\underset{d}{d} \\
\stackrel{d}{a}\end{array}$ & $\frac{\widehat{I}}{\stackrel{J}{E}}$ & 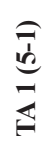 & 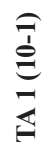 & 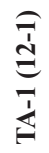 & 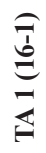 \\
\hline
\end{tabular}

\begin{tabular}{|c|c|c|c|c|c|c|c|}
\hline$\partial^{\circ} \stackrel{\mathscr{n}}{=}$ & $r$ & - & 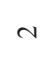 & 6 & 0 & 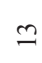 & $?$ \\
\hline H & $m$ & $\hat{n}$ & $\widehat{6}$ & กี & $\ddot{\lambda}$ & $\ddot{\sim}$ & $\stackrel{\infty}{\infty}$ \\
\hline है & $\stackrel{\Xi}{\bar{v}}$ & $\stackrel{n}{\sim}$ & $\overrightarrow{\widetilde{N}}$ & $\stackrel{\beth}{\vec{N}}$ & बें & 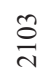 & $\tilde{\overbrace{}}$ \\
\hline H & F & f & 흐 & $F$ & $\hat{n}$ & m & $\stackrel{\infty}{\infty}$ \\
\hline 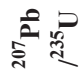 & $\vec{d}$ & $\widehat{\vec{\sim}}$ & ठั & ج্ণ & ڤ્సે & 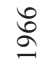 & $\overrightarrow{\widetilde{N}}$ \\
\hline H & $\hat{\imath}$ & ते & 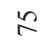 & $\leadsto$ & নे & $\tilde{\lambda}$ & $\tilde{\lambda}$ \\
\hline 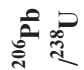 & $\begin{array}{l}\infty \\
\stackrel{\circ}{\circ}\end{array}$ & $\stackrel{\vec{\nu}}{\vec{\lambda}}$ & $\overrightarrow{\hat{~}}$ & $\stackrel{\circ}{\stackrel{\circ}{\sigma}}$ & ठे̀े & $\stackrel{\infty}{\infty}$ & $\stackrel{\partial}{\frac{N}{2}}$ \\
\hline 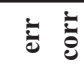 & $\begin{array}{l}0 \\
:\end{array}$ & $\begin{array}{l}3 \\
0 \\
0\end{array}$ & $\stackrel{n}{0}$ & $\begin{array}{l}n \\
0 \\
0\end{array}$ & $\stackrel{\infty}{\stackrel{0}{0}}$ & $\stackrel{n}{0}$ & $\frac{7}{6}$ \\
\hline$\partial^{\circ} \bar{t}$ & $\stackrel{m}{\rightarrow}$ & ले & 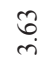 & $\stackrel{\infty}{\stackrel{\sim}{\longrightarrow}}$ & 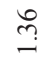 & $\stackrel{\overbrace{}}{\stackrel{2}{\sim}}$ & $\stackrel{\overbrace{}}{\circ}$ \\
\hline 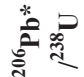 & î & $\begin{array}{l}\text { aे } \\
\stackrel{2}{0}\end{array}$ & ڤ్ & $\begin{array}{l}\overrightarrow{0} \\
\stackrel{0}{0}\end{array}$ & 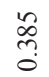 & ले & $\begin{array}{c}\hat{\infty} \\
0 \\
0\end{array}$ \\
\hline$a^{\circ} \bar{c}$ & $\stackrel{\vec{o}}{i}$ & స్ & $\stackrel{\infty}{\infty}$ & $\stackrel{\infty}{\stackrel{\leftrightarrow}{-}}$ & $\stackrel{n}{\stackrel{2}{g}}$ & 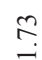 & $\begin{array}{l}\infty \\
\infty \\
\infty\end{array}$ \\
\hline 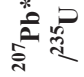 & $\begin{array}{l}\text { ơ } \\
\text { f. }\end{array}$ & $\underset{\pi}{\stackrel{\pi}{\pi}}$ & $\begin{array}{l}\infty \\
\infty \\
\infty \\
0 \\
0\end{array}$ & तี & ڤ్̊ి & $\overrightarrow{\tilde{\sigma}}$ & 总 \\
\hline$\partial^{\circ} \bar{c}$ & 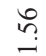 & $\stackrel{?}{\longrightarrow}$ & $\underset{m}{\stackrel{\infty}{m}}$ & $\vec{n}$ & $\stackrel{\varrho}{=}$ & $\stackrel{\infty}{\leftrightarrows}$ & $\tilde{\alpha}$ \\
\hline 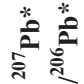 & $\underset{m}{\frac{\pi}{0}}$ & $\begin{array}{l}\infty \\
\stackrel{\pi}{0} \\
0\end{array}$ & $\frac{\vec{m}}{0}$ & $\frac{0}{m}$ & $\begin{array}{l}\overrightarrow{0} \\
\stackrel{0}{0}\end{array}$ & 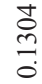 & $\begin{array}{l}\stackrel{0}{0} \\
\stackrel{0}{0}\end{array}$ \\
\hline$\partial^{\circ} \bar{c}$ & $\stackrel{\overbrace{}}{-}$ & ले & $\stackrel{B}{0}$ & $\stackrel{\infty}{\stackrel{\infty}{ }}$ & $\stackrel{\circ}{\stackrel{2}{n}}$ & 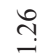 & $\stackrel{\tilde{s}}{\mathrm{r}}$ \\
\hline 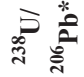 & $\begin{array}{l}\stackrel{0}{\infty} \\
i\end{array}$ & $\stackrel{n}{i}$ & $\underset{i}{\mathbb{d}}$ & $\stackrel{\hat{i}}{i}$ & $\begin{array}{l}\stackrel{8}{0} \\
i\end{array}$ & $\stackrel{\tilde{r}}{\dot{m}}$ & $\begin{array}{c}\infty \\
i \\
i\end{array}$ \\
\hline 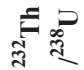 & f․ & లి & ڤn & $\frac{9}{0}$ & $\stackrel{m}{0}$ & ले & $\stackrel{?}{0}$ \\
\hline ֻั้ & $\begin{array}{l}\text { ठे } \\
\text { : }\end{array}$ & $\begin{array}{l}\overline{\tilde{o}} \\
\dot{0}\end{array}$ & तิ & $\stackrel{m}{\circ}$ & 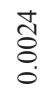 & ठे & 官 \\
\hline 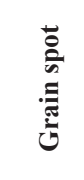 & 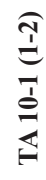 & 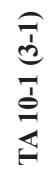 & 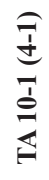 & 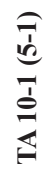 & 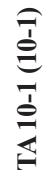 & 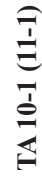 & 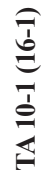 \\
\hline
\end{tabular}

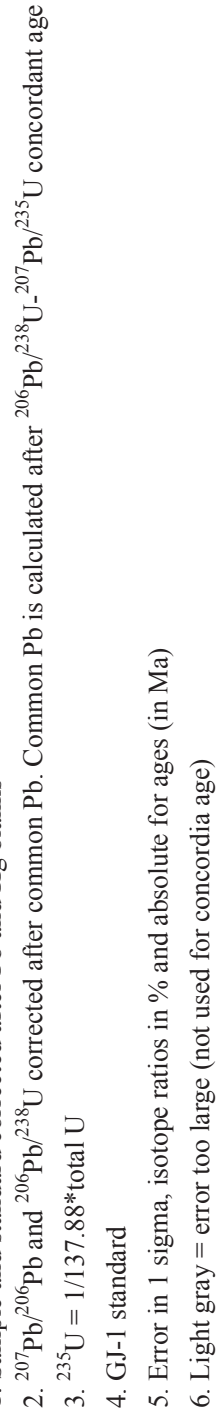




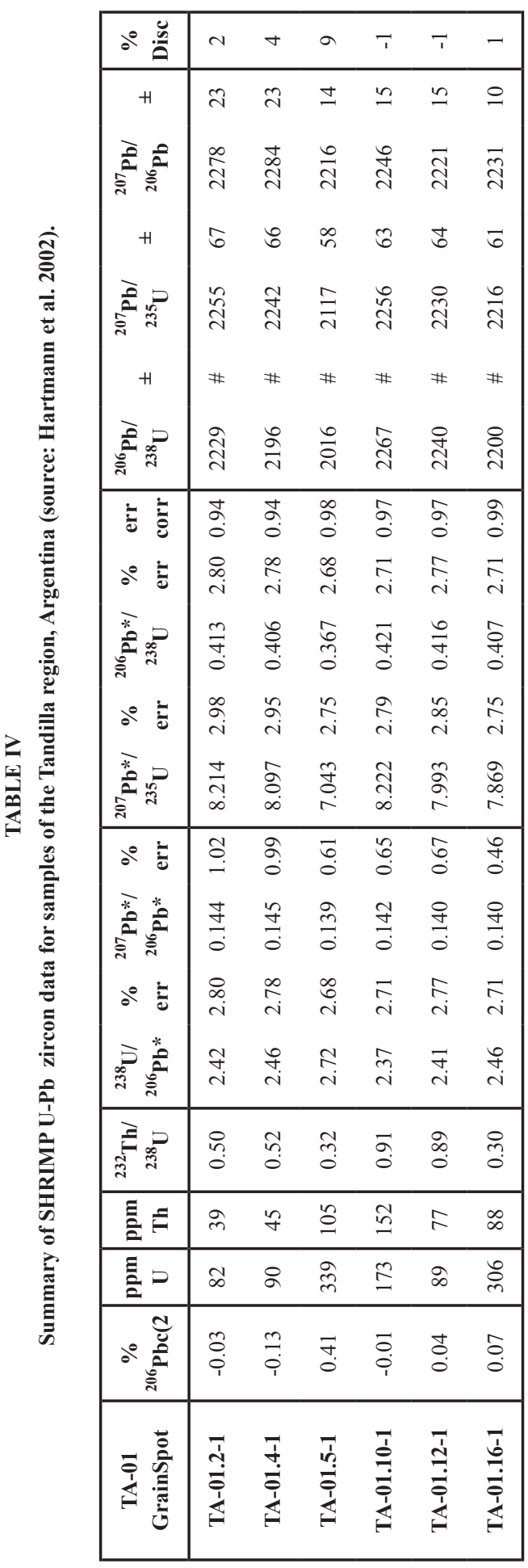

\begin{tabular}{|c|c|c|c|c|c|c|c|c|c|}
\hline$\overbrace{0}^{\circ}$ & $T$ & 0 & $r$ & 0 & 은 & $r$ & $m$ & n & $r$ \\
\hline H & 으 & $\theta$ & $=$ & 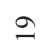 & 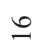 & $a$ & $\infty$ & $=$ & $\infty$ \\
\hline 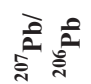 & $\stackrel{\hat{a}}{\sim}$ & 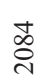 & $\stackrel{\infty}{\stackrel{\text { సे }}{ }}$ & $\underset{⿱}{\stackrel{d}{v}}$ & $\underset{\sim}{\approx}$ & ठ્ेે & $\stackrel{\sim}{\sim}$ & $\stackrel{n}{=}$ & $\stackrel{\infty}{\stackrel{\sim}{े}}$ \\
\hline H & $\hat{\imath}$ & $\bar{m}$ & ֻ & $m$ & $\stackrel{\infty}{\sim}$ & $\ddot{\sim}$ & $\ddot{i n}$ & $\grave{\lambda}$ & $\approx$ \\
\hline Dे & 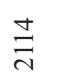 & $\stackrel{\text { वे }}{\text { ते }}$ & ڤ్రి & $\frac{\tilde{\sigma}}{N}$ & 莺 & 岕 & \&̊ & ণ్రి & $\stackrel{m}{\tilde{\Xi}}$ \\
\hline H & $\#$ & \# & \# & \# & \# & \# & $\#$ & \# & \# \\
\hline 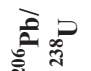 & $\overrightarrow{\widetilde{N}}$ & वे & $\stackrel{8}{\circ}$ & $\underset{\mathrm{\sigma}}{\sim}$ & $\underset{\infty}{\infty}$ & $\stackrel{a}{a}$ & ํํㅇ & ठे & I \\
\hline$\overline{\mathrm{c}}$ & $\hat{\sigma}$ & $\begin{array}{l}\infty \\
\infty \\
0 \\
0\end{array}$ & \& & + & I & + & $\stackrel{n}{o}$ & 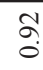 & $\stackrel{n}{o}$ \\
\hline$\partial^{\circ} \bar{c}$ & $\stackrel{\sim}{\sim}$ & $\stackrel{m}{\rightarrow}$ & $\exists$ & $\stackrel{+}{\sim}$ & $\stackrel{\text { I }}{\neg}$ & $\stackrel{\sim}{\sim}$ & $=$ & $\stackrel{\Upsilon}{3}$ & 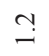 \\
\hline 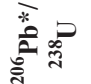 & ळે & 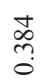 & $\begin{array}{l}n \\
\tilde{n} \\
0\end{array}$ & $\begin{array}{l}n \\
\infty \\
0 \\
0\end{array}$ & है & $\begin{array}{l}n \\
n \\
n \\
0\end{array}$ & 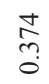 & $\begin{array}{l}\stackrel{0}{0} \\
\stackrel{0}{0}\end{array}$ & $\begin{array}{l}\text { तै } \\
\text { ?. } \\
0\end{array}$ \\
\hline$\partial^{0} \bar{c}$ & $\stackrel{m}{-}$ & $\stackrel{n}{\rightarrow}$ & $\stackrel{?}{-}$ & 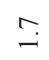 & $\stackrel{ \pm}{-}$ & $\cong$ & 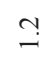 & $\stackrel{m}{-}$ & $\stackrel{\text { I }}{\neg}$ \\
\hline 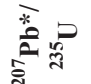 & $\stackrel{\vec{\partial}}{\stackrel{r}{r}}$ & 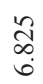 & 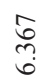 & ๙ૂ & $\begin{array}{l}\text { oे } \\
\text { id } \\
\text { in }\end{array}$ & $\underset{\substack{0 \\
ٍ ్ ర}}{0}$ & 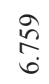 & 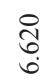 & $\begin{array}{l}\text { ָु } \\
\text { ָु }\end{array}$ \\
\hline do & $n$ & $\hat{o}$ & $\because$ & o. & $\stackrel{\infty}{\circ}$ & $\stackrel{0}{\circ}$ & $\stackrel{t}{\circ}$ & $?$ & $\stackrel{t}{\circ}$ \\
\hline 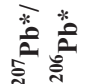 & $\stackrel{\vec{m}}{\stackrel{0}{0}}$ & ते & 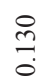 & $\stackrel{0}{0}$ & $\begin{array}{l}\stackrel{\infty}{\Im} \\
\stackrel{0}{0}\end{array}$ & $\underset{0}{0}$ & $\vec{m}$ & $\stackrel{m}{0}$ & 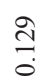 \\
\hline$d^{\circ} \bar{c}$ & 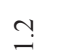 & $\stackrel{?}{\rightarrow}$ & $\exists$ & $\stackrel{+}{-}$ & 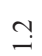 & 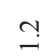 & $=$ & $\stackrel{\sim}{\sim}$ & $\stackrel{\Upsilon}{\longrightarrow}$ \\
\hline 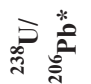 & $\hat{i n}$ & $\underset{\vec{i}}{\vec{i}}$ & $\begin{array}{l}\vec{\infty} \\
i\end{array}$ & in & $\overrightarrow{\dot{m}}$ & $\underset{\infty}{\infty}$ & 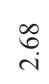 & $\stackrel{\overbrace{}}{i}$ & 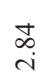 \\
\hline 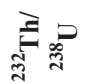 & $\stackrel{n}{\stackrel{0}{o}}$ & ?ึ? & $\stackrel{m}{m}$ & $\begin{array}{c}m \\
\infty \\
0 \\
0\end{array}$ & I & ㅇ․ & $\stackrel{\overbrace{}}{\circ}$ & in & గొ \\
\hline 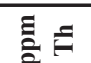 & $\stackrel{m}{\Leftrightarrow}$ & in & 6 & in & 8 & $\stackrel{m}{=}$ & $\bar{\Xi}$ & $\infty$ & 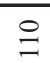 \\
\hline 音口 & $\stackrel{\infty}{\infty}$ & 으 & $m$ & 咅 & 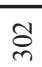 & $\stackrel{\text { }}{\circ}$ & $n$ & 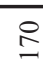 & $\overline{\mathrm{m}}$ \\
\hline 总 & $\stackrel{\circ}{\circ}$ & $\stackrel{\infty}{0}$ & 守 & $\stackrel{0}{\circ}$ & F. & o. & $\stackrel{\theta}{0}$ & 8 & $\stackrel{n}{0}$ \\
\hline 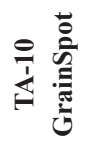 & $\frac{I}{\stackrel{I}{ \pm}}$ & $\frac{\frac{I}{5}}{\frac{1}{3}}$ & $\frac{\bar{j}}{\stackrel{\sigma}{\theta}}$ & $\frac{7}{\stackrel{I}{\oplus}}$ & 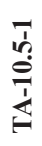 & $\frac{\bar{\sigma}}{\frac{1}{\grave{\theta}}}$ & $\stackrel{I}{\stackrel{I}{\Xi}}$ & $\frac{7}{\frac{7}{9}}$ & $\frac{\bar{b}}{\frac{1}{0}}$ \\
\hline
\end{tabular}

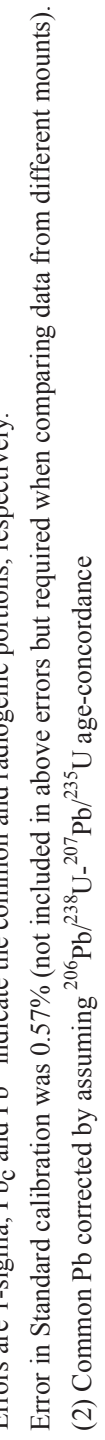


error bar, the most reliable age determination for this rock is the 594 Ma obtained by SHRIMP, as suggested by Babinski et al. (1997). We recognize two zircon phases in the Piquiri Syenite zircons (Fig. 7), where the dated phase is the younger igneous age and interpreted to be the magmatism age of the studied syenitic body.
TEMORA GRABBRODIORITE

Zircons grains of the Temora II, a gabbroic diorite (Australia), have been dated by the LA-MC-ICP-MS method (Table VI). Because the crystallization age of this unit is well constrained by Black et al. (2004) at $416.5 \pm 0.22 \mathrm{Ma}$, the obtained $\mathrm{U}-\mathrm{Pb}$ ratios yielded an
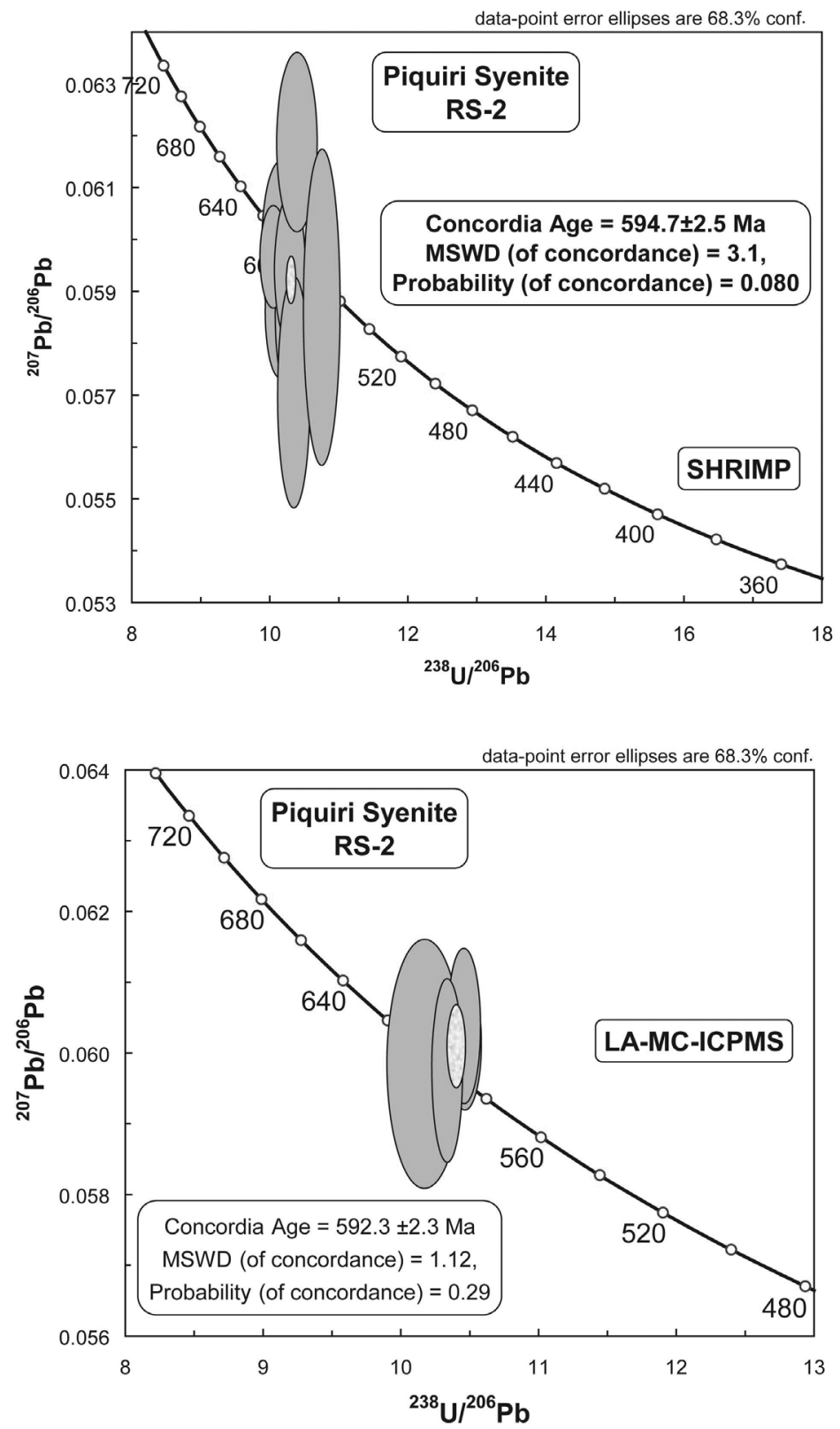

Figure 6. Concordia diagrams for the Piquiri Syenite (Rio Grande do Sul, Brazil) obtained by SHRIMP and LA-MC-ICP-MS. 


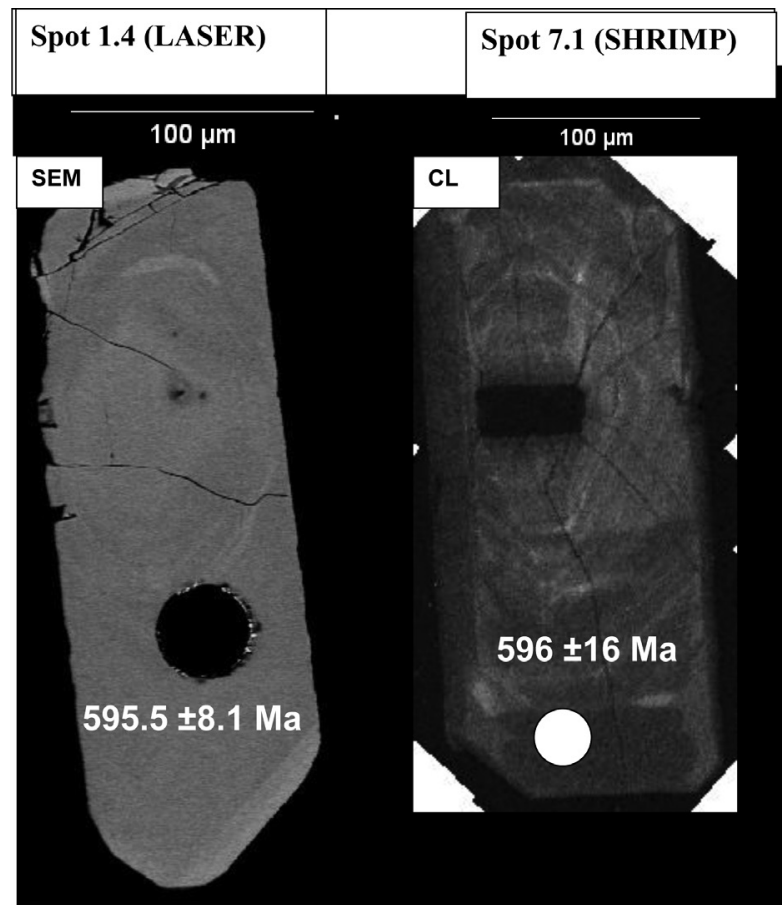

Figure 7. Two different zircon grains dated by LA-MC-ICP-MS and SHRIMP of the Piquiri Syenite (spot size $=25 \mu \mathrm{m})$.

TABLE V

Summary of SHRIMP U-Pb and LA-MC-ICP-MS zircon data for sample RS-2 (Piquiri Syenite, Rio Grande do Sul, Brazil).

\begin{tabular}{|c|c|c|c|c|c|c|c|c|c|c|c|c|c|c|c|c|c|c|c|c|c|}
\hline $\begin{array}{l}\text { SHRIMP } \\
\text { GrainSpot }\end{array}$ & $\begin{array}{c}\% \\
{ }^{206} \mathrm{~Pb}_{\mathrm{c}}(2)\end{array}$ & $\begin{array}{c}\text { ppm } \\
\text { U }\end{array}$ & $\begin{array}{c}\text { ppm } \\
\text { Th }\end{array}$ & $\begin{array}{l}{ }^{232} \mathrm{Th} \\
{ }^{238} \mathrm{U}\end{array}$ & $\begin{array}{c}\mathrm{ppm} \\
{ }^{206} \mathrm{~Pb}^{*}\end{array}$ & $\begin{array}{c}{ }^{238} \mathrm{U} / \\
{ }^{206} \mathrm{~Pb}^{*}\end{array}$ & $\begin{array}{l}\% \\
\text { err }\end{array}$ & $\begin{array}{l}{ }^{207} \mathrm{~Pb}^{*} \\
\rho^{206} \mathrm{~Pb}^{*}\end{array}$ & $\begin{array}{c}\% \\
\text { err }\end{array}$ & $\begin{array}{c}{ }^{207} \mathrm{~Pb}^{*} \\
{ }^{235} \mathrm{U}\end{array}$ & $\begin{array}{c}\% \\
\text { err }\end{array}$ & $\begin{array}{c}{ }^{206} \mathbf{P b}^{*} \\
{ }^{238} \mathrm{U}\end{array}$ & $\begin{array}{l}\% \\
\text { err }\end{array}$ & $\begin{array}{l}\text { err } \\
\text { corr }\end{array}$ & $\begin{array}{l}{ }^{206} \mathrm{~Pb} \\
{ }^{238} \mathrm{U}\end{array}$ & \pm & $\begin{array}{l}{ }^{207} \mathrm{~Pb} \\
{ }^{235} \mathrm{U}\end{array}$ & \pm & $\begin{array}{l}{ }^{207} \mathrm{~Pb} \\
\rho^{206} \mathrm{~Pb}\end{array}$ & \pm & $\begin{array}{c}\% \\
\text { Disc }\end{array}$ \\
\hline 1.1 & 0.17 & 270 & 171 & 0.66 & 22.2 & 10.47 & 1.3 & .0586 & 1.4 & 0.771 & 1.9 & 0.095 & 1.3 & .694 & 588 & 8 & 581 & 11 & 552 & 8 & -7 \\
\hline 3.1 & 0.17 & 138 & 160 & 1.20 & 11.7 & 10.19 & 1.8 & .0598 & 1.9 & 0.809 & 2.6 & 0.098 & 1.8 & .692 & 604 & 11 & 602 & 16 & 597 & 11 & -1 \\
\hline 4.1 & 0.18 & 155 & 111 & 0.74 & 13.0 & 10.26 & 1.4 & .0588 & 1.9 & 0.790 & 2.4 & 0.098 & 1.4 & .602 & 600 & 9 & 591 & 14 & 560 & 11 & -7 \\
\hline 5.1 & 0.00 & 195 & 140 & 0.74 & 16.5 & 10.17 & 1.4 & .0586 & 1.5 & 0.795 & 2.0 & 0.098 & 1.4 & .685 & 605 & 8 & 594 & 12 & 554 & 8 & -9 \\
\hline 5.2 & 0.24 & 227 & 154 & 0.70 & 18.9 & 10.31 & 1.4 & .0585 & 1.7 & 0.783 & 2.2 & 0.097 & 1.4 & .616 & 597 & 8 & 587 & 13 & 550 & 10 & -9 \\
\hline 6.1 & 0.00 & 496 & 647 & 1.35 & 42.3 & 10.07 & 1.3 & .0597 & 1.1 & 0.817 & 1.7 & 0.099 & 1.3 & .756 & 610 & 8 & 606 & 10 & 592 & 7 & -3 \\
\hline 6.2 & 0.56 & 475 & 242 & 0.53 & 39.0 & 10.51 & 1.5 & .0594 & 1.9 & 0.779 & 2.4 & 0.095 & 1.5 & .620 & 586 & 9 & 585 & 14 & 583 & 11 & -1 \\
\hline 7.1 & 0.00 & 166 & 191 & 1.19 & 13.8 & 10.31 & 1.4 & .0594 & 1.6 & 0.795 & 2.1 & 0.097 & 1.4 & .667 & 597 & 9 & 594 & 13 & 583 & 9 & -2 \\
\hline 8.1 & 0.27 & 142 & 104 & 0.76 & 11.8 & 10.37 & 1.5 & .0571 & 2.6 & 0.758 & 3.0 & 0.096 & 1.5 & .497 & 593 & 9 & 573 & 17 & 494 & 13 & -20 \\
\hline 9.1 & 0.00 & 119 & 100 & 0.86 & 9.84 & 10.41 & 1.9 & .0619 & 1.9 & 0.820 & 2.6 & 0.096 & 1.9 & .713 & 591 & 11 & 608 & 16 & 671 & 12 & 12 \\
\hline 10.1 & 0.51 & 189 & 175 & 0.96 & 15.1 & 10.77 & 1.6 & .0587 & 3.4 & 0.752 & 3.8 & 0.093 & 1.6 & .425 & 572 & 9 & 569 & 22 & 556 & 19 & -3 \\
\hline
\end{tabular}

${ }^{1}$ Samples and standards corrected after blank and $\mathrm{Hg}$

${ }^{2}$ 207/206 and 206/238 ratios corrected after common ${ }^{204} \mathrm{~Pb}$. Common $\mathrm{Pb}$ corrected assuming ${ }^{206} \mathrm{~Pb} /{ }^{238} \mathrm{U}-{ }^{235} \mathrm{~Pb} /{ }^{235} \mathrm{U}$ age-concordance ${ }^{3} 235 \mathrm{U}=1 / 137.88^{*}$ Utotal

${ }^{4}$ Standard GJ-1 
FARID CHEMALE Jr et al.

TABLE V

(continuation)

\begin{tabular}{|c|c|c|c|c|c|c|c|c|c|c|c|c|c|c|c|c|c|c|}
\hline $\begin{array}{c}\text { LA-MC- } \\
\text { ICPMS } \\
\text { GrainSpot }\end{array}$ & $\%{ }^{206} \mathrm{~Pb}_{\mathrm{c}}(2)$ & $\begin{array}{c}\mathbf{h} \\
\rho^{238} \mathbf{U}\end{array}$ & $\begin{array}{c}{ }^{238} \mathrm{U} / \\
{ }^{206} \mathrm{~Pb}^{*}\end{array}$ & $\begin{array}{c}\% \\
\text { err }\end{array}$ & $\begin{array}{l}{ }^{207} \mathrm{~Pb}^{*} \\
{ }^{206} \mathrm{~Pb}^{*}\end{array}$ & $\begin{array}{l}\% \\
\text { err }\end{array}$ & $\begin{array}{c}{ }^{207} \mathbf{P b}^{*} \\
{ }^{235} \mathrm{U}\end{array}$ & $\begin{array}{c}\% \\
\text { err }\end{array}$ & $\begin{array}{l}{ }^{206} \mathbf{P b}^{*} \\
{ }^{238} \mathrm{U}\end{array}$ & $\begin{array}{c}\% \\
\text { err }\end{array}$ & $\begin{array}{l}\text { err } \\
\text { corr }\end{array}$ & $\begin{array}{l}{ }^{206} \mathrm{~Pb} \\
{ }^{238} \mathrm{U}\end{array}$ & \pm & $\begin{array}{c}{ }^{207} \mathbf{P b} \\
/^{235}\end{array}$ & \pm & $\begin{array}{c}{ }^{207} \mathrm{~Pb} \\
2^{206}\end{array}$ & \pm & $\begin{array}{c}\% \\
\text { Disc }\end{array}$ \\
\hline 1.1 & 0.06 & $\mathrm{n} / \mathrm{d}$ & 10.45 & 0.8 & .0604 & 1.2 & 0.796 & 1.4 & 0.096 & 0.8 & .531 & 589 & 4 & 595 & 8 & 617 & 7 & 5 \\
\hline 1.11 & 0.10 & $\mathrm{n} / \mathrm{d}$ & 10.46 & 0.8 & .0601 & 1.3 & 0.793 & 1.3 & 0.096 & 0.8 & .591 & 589 & 4 & 593 & 8 & 609 & 8 & 3 \\
\hline 1.4 & 0.25 & 0.66 & 10.33 & 0.7 & .0598 & 1.4 & 0.797 & 1.6 & 0.097 & 0.7 & .446 & 596 & 4 & 595 & 10 & 595 & 9 & 0 \\
\hline 1.8 & 0.06 & 1.24 & 10.17 & 1.8 & .0599 & 1.0 & 0.812 & 2.6 & 0.098 & 1.8 & .677 & 605 & 11 & 603 & 16 & 598 & 6 & -1 \\
\hline
\end{tabular}

1 Samples and standards corrected after blank and $\mathrm{Hg}$

2 207/206 and 206/238 ratios corrected after common ${ }^{204} \mathrm{~Pb}$. Common Pb corrected assuming ${ }^{206} \mathrm{~Pb} /{ }^{238} \mathrm{U}_{-}{ }^{235} \mathrm{~Pb} /{ }^{235} \mathrm{U}$ age-concordance $3235 \mathrm{U}=1 / 137.88 *$ Utotal

4 Standard GJ-1

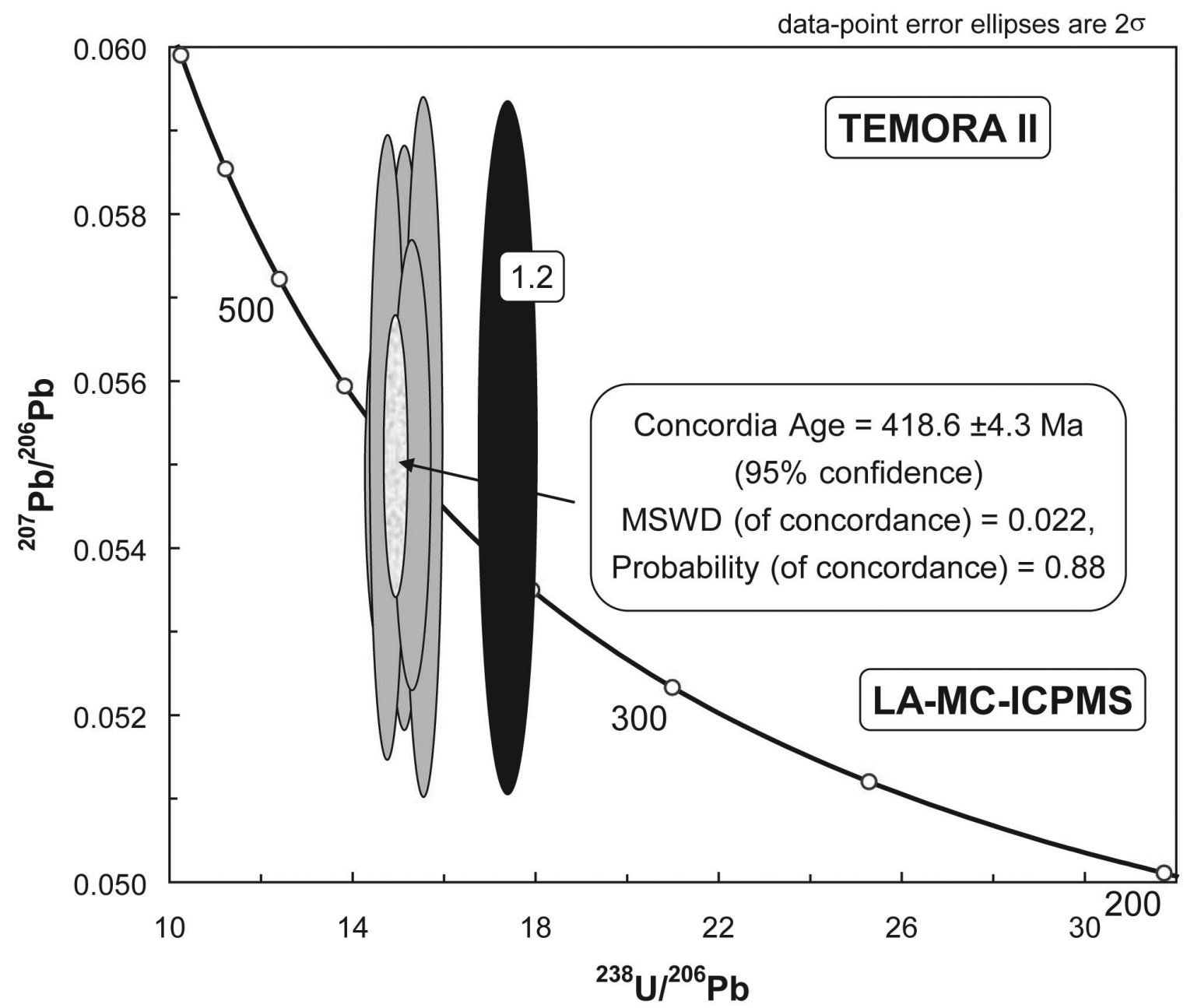

Figure 8. Concordia diagrams with LA-MC-ICP-MS results for sample of Temora II (Australia). 
TABLE VI

Summary of LA-MC-ICP-MS U-Pb zircon data for sample Temora II (Australia).

\begin{tabular}{|c|c|c|c|c|c|c|c|c|c|c|c|c|c|c|c|c|c|c|}
\hline $\begin{array}{c}\text { Grain } \\
\text { spot }\end{array}$ & f206 & $\begin{array}{l}{ }^{232} \mathrm{Th} \\
{ }^{238} \mathrm{U}\end{array}$ & $\begin{array}{c}{ }^{238} \mathrm{U} / \\
{ }^{206} \mathrm{~Pb}^{*}\end{array}$ & $\begin{array}{c}\% \\
\text { err }\end{array}$ & $\begin{array}{l}{ }^{207} \mathrm{~Pb}^{*} \\
{ }^{206} \mathrm{~Pb}^{*}\end{array}$ & $\begin{array}{c}\% \\
\text { err }\end{array}$ & $\begin{array}{c}{ }^{207} \mathbf{P b}^{*} \\
{ }^{235} \mathbf{U}\end{array}$ & $\begin{array}{c}\% \\
\text { err }\end{array}$ & $\begin{array}{c}{ }^{206} \mathrm{~Pb}^{*} \\
{ }^{238} \mathrm{U}\end{array}$ & $\begin{array}{c}\% \\
\text { err }\end{array}$ & $\begin{array}{c}\text { err } \\
\text { corr }\end{array}$ & $\begin{array}{r}{ }^{206} \mathrm{~Pb} \\
{ }^{238} \mathrm{U}\end{array}$ & \pm & $\begin{array}{l}{ }^{207} \mathrm{~Pb} \\
{ }^{235} \mathrm{U}\end{array}$ & \pm & $\begin{array}{r}{ }^{207} \mathrm{~Pb} \\
{ }^{206} \mathrm{~Pb}\end{array}$ & \pm & $\begin{array}{c}\% \\
\text { Disc }\end{array}$ \\
\hline 1.1 & 0.0006 & 0.25 & 14.77 & 1.07 & 0.0552 & 1.50 & 0.516 & 1.84 & 0.068 & 1.07 & 0.58 & 422 & 5 & 422 & 8 & 422 & 6 & 0 \\
\hline 1.2 & 0.0006 & 0.46 & 17.32 & 1.48 & 0.0552 & 3.11 & 0.439 & 3.45 & 0.058 & 1.48 & 0.43 & 362 & 5 & 370 & \# & 370 & \# & 14 \\
\hline 1.3 & 0.0010 & 0.20 & 15.04 & 1.10 & 0.0553 & 1.34 & 0.507 & 2.85 & 0.66 & 1.10 & 0.39 & 415 & 5 & 416 & \# & 416 & 6 & 2 \\
\hline 1.4 & 0.0011 & 0.28 & 15.48 & 1.12 & 0.0552 & 1.70 & 0.492 & 3.33 & 0.065 & 1.12 & 0.34 & 404 & 5 & 406 & \# & 406 & 7 & 4 \\
\hline 1.5 & 0.0005 & 0.37 & 14.56 & 1.05 & 0.0548 & 1.60 & 0.519 & 1.92 & 0.069 & 1.05 & 0.55 & 428 & 5 & 424 & 8 & 424 & 6 & -6 \\
\hline 1.5 & 0.0009 & 0.37 & 15.00 & 1.08 & 0.0550 & 1.69 & 0.506 & 2.01 & 0.067 & 1.08 & 0.54 & 416 & 5 & 416 & 8 & 416 & 7 & -1 \\
\hline 1.7 & 0.0004 & 0.28 & 14.68 & 1.06 & 0.0554 & 2.21 & 0.520 & 2.46 & 0.068 & 1.06 & 0.43 & 425 & 5 & 425 & \# & 425 & 9 & 1 \\
\hline 1.8 & 0.0001 & 0.35 & 14.67 & 1.06 & 0.0552 & 2.80 & 0.519 & 3.00 & 0.068 & 1.06 & 0.35 & 425 & 5 & 424 & \# & 424 & \# & -1 \\
\hline 1.9 & 0.0006 & 0.42 & 15.23 & 1.10 & 0.0550 & 2.03 & 0.498 & 2.31 & 0.066 & 1.10 & 0.48 & 410 & 5 & 410 & 9 & 410 & 8 & 0 \\
\hline
\end{tabular}

1. Sample and standard corrected after $\mathrm{Pb}$ and $\mathrm{Hg}$ blanks

2. ${ }^{207} \mathrm{~Pb} /{ }^{206} \mathrm{~Pb}$ and ${ }^{206} \mathrm{~Pb} /{ }^{238} \mathrm{U}$ corrected after common $\mathrm{Pb}$. Common $\mathrm{Pb}$ is calculated after ${ }^{206} \mathrm{~Pb} /{ }^{238} \mathrm{U}-{ }^{207} \mathrm{~Pb} /{ }^{235} \mathrm{U}$ concordant age

3. ${ }^{235} \mathrm{U}=1 / 137.88 *$ total $\mathrm{U}$

4. GJ-1 standard

5. Error in 1 sigma, isotope ratios in \% and absolute for ages (in $\mathrm{Ma}$ )

6 . Light gray $=$ discordant (not used for concordia age)

age of 418.6 $\pm 4.3 \mathrm{Ma}$ (Fig. 8), which is close to the IDTIMS data. However, because the zircons of Temora II have little $\mathrm{Pb} 207$, we had to use a spot size of 40 $\mu \mathrm{m}$ to obtain reliable isotopic data. The dated zircon grains are highly homogeneous and inclusion-free.

\section{TRIASSIC VOLCANIC ROCKS}

A rhyolite (sample RB-06) is situated at the basal portion of the Rincon Blanco Basin and located in the Precordillera fold and thrust belt at $31^{\circ} 24^{\prime}-31^{\circ} 33^{\prime}$ south. A dating of the youngest zircons of this sample yielded ages of 244.1 $\pm 3.5 \mathrm{Ma}$ (LA-MC-ICP-MS) and 246.4 $\pm 1.1 \mathrm{Ma}$ (SHRIMP, Barredo et al. 2012) (Fig. 9 and tables VII and VIII), which are coeval to those basal rhyolites of the Cuyo Basin (Ávila et al. 2006), a contemporaneous volcano-sedimentary basin of the Triassic Intracontinental rifting exposed in the Precordillera region, Argentina.
The Triassic sample was mostly dated with a $40 \mu \mathrm{m}$ spot size with the laser method because the ${ }^{207} \mathrm{~Pb}^{*}$ content was lower than 5,000 cps using a spot size of $25 \mu \mathrm{m}$. In this case, the analytical data presents high statistical errors, and the ratios cannot be estimated precisely. Figures 10 and 9 present $\mathrm{Cl}, \mathrm{BSE}$ and reflected light (RE) images of dated zircons as well as the calculated individual ages for the spots obtained by SHRIMP and Laser. It is noteworthy that the age errors by the Laser method are larger than those obtained by the SHRIMP method for the same zircon phases (see Figure 10).

\section{DISCUSSION}

The analytical strategies using laser ablation coupled to an ICP-MS instrument vary according to certain parameters that must be taken into account in the available system. As a result, 

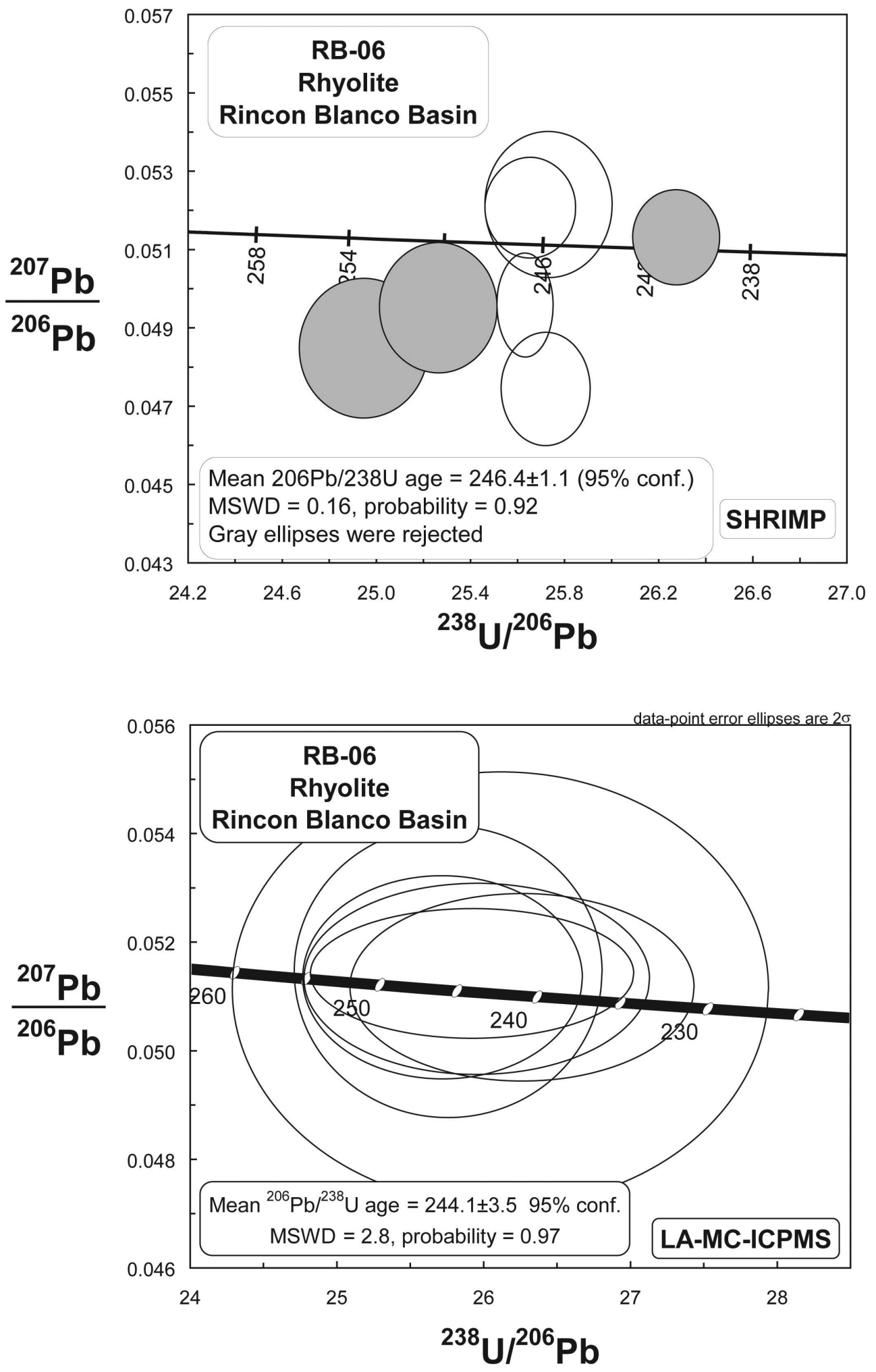

Figure 9. Concordia diagrams with SHRIMP (Barredo et al. 2012) and LA-MC-ICP-MS results for RB-06 sample of the Rincon Blanco basin (Argentina). 

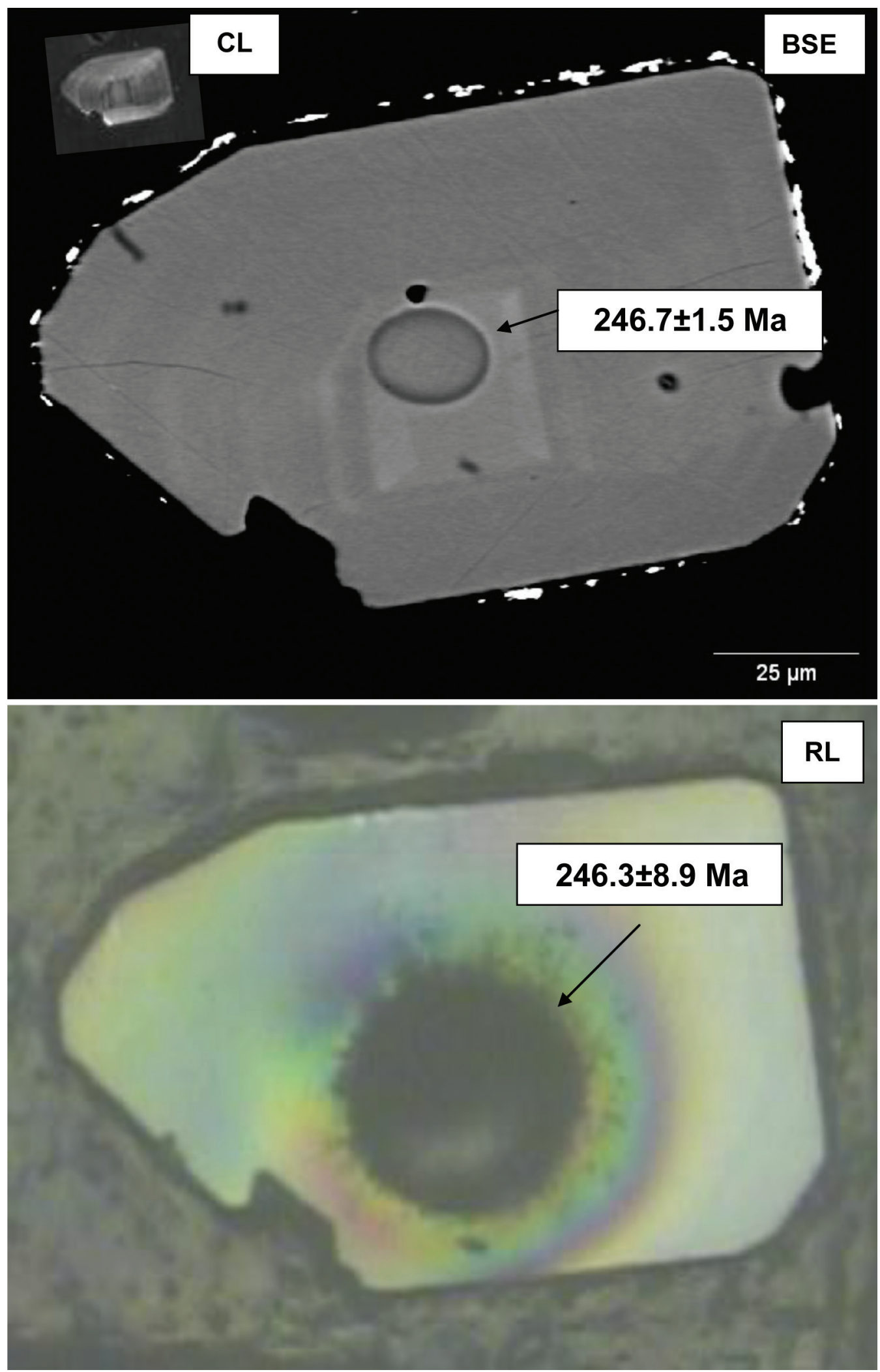

Figure 10. RB-06 -04 grain with the SHRIMP spot of ca. $20 \mu \mathrm{m}$ (SEM) and Laser spot of $40 \mu \mathrm{m}$ (see text for explanation) 
TABLE VII

Summary of LA-MC-ICP-MS U-Pb zircon data for sample RB-06 of the Rincon Blanco basin, Argentina.

\begin{tabular}{|c|c|c|c|c|c|c|c|c|c|c|c|c|c|c|c|c|c|c|}
\hline Grain spot & f206 & $\begin{array}{l}{ }^{232} \mathrm{Th} \\
f^{238} \mathrm{U}\end{array}$ & ${ }^{238}{ }^{206} \mathrm{~Pb}^{*}$ & $\begin{array}{c}\% \\
\text { err }\end{array}$ & $\begin{array}{l}{ }^{207} \mathbf{P b}^{*} \\
/^{206} \mathbf{P b}^{*}\end{array}$ & $\begin{array}{c}\% \\
\text { err }\end{array}$ & $\begin{array}{c}{ }^{207} \mathbf{P b}^{*} \\
{ }^{235} \mathbf{U}\end{array}$ & $\begin{array}{c}\% \\
\text { err }\end{array}$ & $\begin{array}{c}{ }^{206} \mathbf{P b}^{*} \\
{ }^{238} \mathbf{U}\end{array}$ & $\begin{array}{c}\% \\
\text { err }\end{array}$ & $\begin{array}{c}\text { err } \\
\text { corr }\end{array}$ & $\begin{array}{l}{ }^{206} \mathrm{~Pb} \\
{ }^{238} \mathrm{U}\end{array}$ & \pm & $\begin{array}{r}{ }^{207} \mathrm{~Pb} \\
{ }^{235} \mathrm{U}\end{array}$ & \pm & $\begin{array}{r}{ }^{207} \mathrm{~Pb} \\
{ }^{206} \mathrm{~Pb}\end{array}$ & \pm & $\begin{array}{c}\% \\
\text { Disc }\end{array}$ \\
\hline RB06-03 & 0.0013 & 0.65 & 25,75 & 1.67 & 0.0514 & 2.15 & 0.275 & 2.72 & 0.039 & 1.67 & 0.61 & 246 & 4 & 247 & 7 & 261 & 6 & 6 \\
\hline RB06-04 & 0.0003 & 0.86 & 25.95 & 1.86 & 0.0513 & 1.41 & 0.273 & 2.33 & 0.039 & 1.86 & 0.80 & 244 & 5 & 245 & 6 & 255 & 4 & 4 \\
\hline RB06-05 & 0.0000 & 0.87 & 26.26 & 1.83 & 0.0512 & 1.38 & 0.269 & 2.30 & 0.038 & 1.83 & 0.80 & 241 & 4 & 242 & 6 & 248 & 3 & 3 \\
\hline RB06-10 & 0.0005 & 1.30 & 24.88 & 1.62 & 0.0509 & 3.38 & 0.282 & 3.75 & 0.040 & 1.62 & 0.43 & 254 & 4 & 252 & 9 & 237 & 8 & -7 \\
\hline RB06-15 & 0.0001 & 0.67 & 25.92 & 1.74 & 0.0514 & 0.95 & 0.274 & 1.99 & 0.039 & 1.74 & 0.88 & 244 & 4 & 246 & 5 & 260 & 2 & 6 \\
\hline RB06-16 & 0.0027 & 0.70 & 26.56 & 1.97 & 0.0511 & 3.00 & 0.265 & 3.59 & 0.038 & 1.97 & 0.55 & 238 & 5 & 239 & 9 & 246 & 7 & 3 \\
\hline RB06-19* & 0.0017 & 0.64 & 26.11 & 2.87 & 0.0511 & 3.21 & 0.270 & 4.31 & 0.038 & 2.87 & 0.67 & 242 & 7 & 243 & 10 & 246 & 8 & 2 \\
\hline RB06-20* & 0.0009 & 0.57 & 26.83 & 2.96 & 0.0513 & 2.34 & 0.263 & 3.78 & 0.037 & 2.96 & 0.78 & 236 & 7 & 237 & 9 & 253 & 6 & 7 \\
\hline RB06-26 & 0.4396 & 1.08 & 26.41 & 1.79 & 0.0512 & 3.48 & 0.267 & 3.92 & 0.038 & 1.79 & 0.46 & 240 & 4 & 240 & 9 & 248 & 9 & 4 \\
\hline RB06-27 & 0.0014 & 0.75 & 25.72 & 1.52 & 0.0513 & 1.50 & 0.275 & 2.13 & 0.039 & 1.52 & 0.71 & 246 & 4 & 247 & 5 & 256 & 4 & 4 \\
\hline
\end{tabular}

1. Sample and standard corrected after $\mathrm{Pb}$ and $\mathrm{Hg}$ blanks

2. ${ }^{207} \mathrm{~Pb} /{ }^{206} \mathrm{~Pb}$ and ${ }^{206} \mathrm{~Pb} /{ }^{238} \mathrm{U}$ corrected after common $\mathrm{Pb}$. Common $\mathrm{Pb}$ is calculated after ${ }^{206} \mathrm{~Pb} /{ }^{238} \mathrm{U}-{ }^{207} \mathrm{~Pb} /{ }^{235} \mathrm{U} \mathrm{concordant}$ age

3. ${ }^{235} \mathrm{U}=1 / 137.88 *$ total $\mathrm{U}$

4. GJ-1 standard

5. Error in 1 sigma, isotope ratios in \% and absolute for ages (in Ma)

6. Light gray not included in the age calculation

* Spot size $25 \mu \mathrm{m}$, otherwise the spots were $40 \mu \mathrm{m}$

TABLE VIII

Summary of SHRIMP U-Pb zircon data for sample RB-06 of the Rincon Blanco basin, Argentina (source: Barredo et al. 2012).

\begin{tabular}{|c|c|c|c|c|c|c|c|c|c|c|c|c|c|c|c|c|c|c|c|c|c|}
\hline GrainSpot & ${ }^{206}{ }^{\%}{ }^{\%} b_{c}(2)$ & $\begin{array}{c}\text { ppm } \\
\mathrm{U}\end{array}$ & $\begin{array}{c}\text { ppm } \\
\text { Th }\end{array}$ & 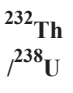 & $\begin{array}{c}\mathrm{ppm} \\
{ }^{206} \mathrm{~Pb}^{*}\end{array}$ & $\begin{array}{c}{ }^{238} \mathrm{U} / \\
{ }^{206} \mathrm{~Pb}^{*}\end{array}$ & $\begin{array}{l}\% \\
\text { err }\end{array}$ & $\begin{array}{l}{ }^{207} \mathrm{~Pb}^{*} \\
{ }^{206} \mathrm{~Pb}^{*}\end{array}$ & $\begin{array}{c}\% \\
\text { err }\end{array}$ & ${ }^{207} \mathbf{P b}^{*}$ & $\begin{array}{c}\% \\
\text { err }\end{array}$ & ${ }^{{ }^{206} \mathbf{P b}^{*}} \mathbf{U}$ & $\begin{array}{c}\% \\
\text { err }\end{array}$ & $\begin{array}{l}\text { err } \\
\text { corr }\end{array}$ & $\begin{array}{l}{ }^{206} \mathbf{P b} \\
{ }^{238} \mathbf{U}\end{array}$ & \pm & $\begin{array}{l}{ }^{207} \mathrm{~Pb} \\
{ }^{235} \mathrm{U}\end{array}$ & \pm & $\begin{array}{l}{ }^{207} \mathrm{~Pb} \\
{ }^{206} \mathrm{~Pb}\end{array}$ & \pm & $\begin{array}{c}\% \\
\text { Disc }\end{array}$ \\
\hline RB06-01 & -0.171 & 437 & 558 & 1.28 & 14.6 & 25.7 & 0.5 & 0.0521 & 1.6 & 0.280 & 1.7 & 0.039 & 0.5 & 0.29 & 246 & 1 & 251 & 4 & 290 & 5 & 15 \\
\hline RB06-03 & 0.000 & 195 & 168 & 0.86 & 6.7 & 25.2 & 0.7 & 0.0490 & 2.8 & 0.268 & 2.5 & 0.040 & 0.7 & 0.29 & 253 & 2 & 241 & 6 & 149 & 4 & -70 \\
\hline RB06-04 & 0.273 & 2010 & 1374 & 0.68 & 67.3 & 25.6 & 0.3 & 0.0496 & 1.8 & 0.267 & 1.8 & 0.039 & 0.3 & 0.17 & 247 & 1 & 240 & 4 & 177 & 3 & -40 \\
\hline RB06-05 & 0.228 & 344 & 201 & 0.58 & 11.5 & 25.7 & 0.5 & 0.0475 & 2.0 & 0.255 & 2.1 & 0.039 & 0.5 & 0.24 & 246 & 1 & 230 & 5 & 73 & 1 & -235 \\
\hline RB06-06 & 0.000 & 202 & 125 & 0.62 & 6.7 & 25.8 & 0.7 & 0.0523 & 2.5 & 0.280 & 2.5 & 0.039 & 0.7 & 0.28 & 246 & 2 & 250 & 6 & 299 & 7 & 18 \\
\hline RB06-07 & 0.000 & 496 & 300 & 0.60 & 16.2 & 26.3 & 0.5 & 0.0513 & 1.5 & 0.269 & 1.6 & 0.038 & 0.5 & 0.29 & 241 & 1 & 242 & 4 & 255 & 4 & 6 \\
\hline RB06-08 & 0.000 & 230 & 172 & 0.75 & 7.8 & 25.4 & 0.7 & 0.0498 & 2.3 & 0.270 & 2.3 & 0.040 & 0.7 & 0.28 & 250 & 2 & 243 & 6 & 184 & 4 & -36 \\
\hline
\end{tabular}

Errors are 1-sigma; $\mathrm{Pbc}$ and $\mathrm{Pb} *$ indicate the common and radiogenic portions, respectively.

Error in Standard calibration was $0.57 \%$ (not included in above errors but required when comparing data from different mounts).

(2) Common $\mathrm{Pb}$ corrected by assuming ${ }^{206} \mathrm{~Pb} /{ }^{238} \mathrm{U}-{ }^{207} \mathrm{~Pb} /{ }^{235} \mathrm{U}$ age-concordance

Spots marked with light gray are not included in the concordia age calculation 
we have no "best" parameters to date zircons. The basic principles in such systems are a good control of the spot size (using CI, BSE and R1 images), the quality of the measurements and adequate fractionation (elemental and isotopic) corrections. Another important feature is to select with well-established criteria the ratios needed to obtain the mean or extrapolated parameters.

The mixed configuration with MC-ICP-MS (Neptune) and the coupled Laser Microprobe (New Wave $213 \mathrm{~nm}$ ) have some advantages for dating zircons of different ages. The ${ }^{207} \mathrm{~Pb}$ mass is therefore collected with a MIC channel, while the ${ }^{206} \mathrm{~Pb},{ }^{232} \mathrm{Th}$ and ${ }^{238} \mathrm{U}$ are collected in the Faraday cups because the amount of ${ }^{207} \mathrm{~Pb}^{*}$ in the zircons, especially those of Neoproterozoic to Phanerozoic ages, is too low to be collected with Faraday cups with the laser conditions applied in this work. However, the obtained values for the ${ }^{206} \mathrm{~Pb},{ }^{232} \mathrm{Th}$ and ${ }^{238} \mathrm{U}$ are from some $\mathrm{mV}$ up to one hundred $\mathrm{mV}$, and uncertainties are less than $1 \%$ for the obtained ratios. In addition, the calculated ratios for ${ }^{238} \mathrm{U} /{ }^{207} \mathrm{~Pb}$ for zircons formed from $1.0 \mathrm{Ga}$ to 20 Ma vary from 80 to 666.67 , which can introduce large uncertainties if we analyze 238 and 207 with the same type of ion collectors. To obtain more precise ratios, we used the suggested mixed configuration in this present work. In the case in which the amount of ${ }^{207} \mathrm{~Pb}$ was to high (over $15 \mathrm{mV}$ or $1,000,000 \mathrm{cps}$ ), we decreased the size of the spot (to $15 \mu \mathrm{m}$ ) or the energy of the laser to maintain the same configuration. Alternatively, we can use different configurations of mixed collectors (masses 202, 204, 206 and 207 in MIC channels and mass 238 in Faraday, Buhn et al. 2009) or with the same type of collector (only MIC's channels or Faraday cups).

The present sample dating supports the idea that the LA-MC-ICP-MS method presented here can also be used for dating igneous rocks. We found that the results are somewhat comparable to the SHRIMP data (see Table I). In broad terms,
SHRIMP is more precise and has a greater accuracy because it does not have the strong fractionation provoked by the laser (e.g., $\mathrm{U}$ and $\mathrm{Pb}$ isotopes). Also, there is no $\mathrm{Hg}$ interference, and it has a much higher resolution (MC-ICP-MS operated here at low resolution (800), while SHRIMP was routinely up to 5,000). Thus, the greater reliability of the SHRIMP data is also due to the size of the analyzed particle because SHRIMP produces a hole no more than $3 \mu \mathrm{m}$ deep for 15 minutes of analysis, while the laser goes up to $15 \mu \mathrm{m}$ or more in ca. 50 seconds. Therefore, the particle size produced by the laser is too large, producing in most cases some variation of the obtained signal, which certainly increases the error estimation and the age of the analyzed zircon (spot). If the laser could produce smaller particles, the obtained data would be much better. This goal may be attainable in the near future using the Fenton Laser (Koesler and Sylvester 2003, Cocherie and Robert 2008).

The presence of large amounts of common $\mathrm{Pb}$ was identified either with SHRIMP or with LAMC-ICP-MS. In this case, the spot data cannot be used. The presence of common $\mathrm{Pb}$ is mostly related to metamictic zircon or the mounting resin. In some cases, the laser produces some instability that results in very few usable cycles for the spots, which makes it difficult to obtain a single spot age and the acquired data not useful at all. Therefore, for dating igneous rocks, it is necessary to have a larger number of zircon spots to obtain comparable results with SHRIMP. Based on our results and comparing the SHRIMP and LA-MC-ICPMS analytical data, we observe that the individual error obtained by LAMC-ICPMS is usually several times larger (3 to 4 times) than those obtained by the SHRIMP method. It is also emphasized that the calculated age based on LA-MC-ICPMS presents an uncertainty over 1 $\%$ (for Phanerozoic rocks, it can be $2 \%$ or more), whereas the SHRIMP age uncertainties are less than $1 \%$. This assumption is valid for homogeneous zircon, as we used in this study. 
The GJ-1 zircon was a very suitable standard with relatively high $U$ content and homogeneous $\mathrm{U}-\mathrm{Th} / \mathrm{Pb}$ ratios, as already presented by Jackson et al. (2004). In our case, we used a large crystal (ca. $36 \mathrm{~mm} 2)$ mounted in a small cylinder $(8 \mathrm{~mm}$ diameter) inserted in the sample mounting which provides consistent analytical data during a session day, even analyzing with $15 \mu \mathrm{m}$ and $25 \mu \mathrm{m}$ spot sizes in the same analytical conditions. In the case of the grain size of the zircon standard, like those used for SHRIMP (Temora II, FC1), the resulting data are much more variable during the Laser analyses due to the small size of the zircon crystal.

\section{CONCLUSIONS}

The analytical data obtained by the LA-MC-ICPMS method using a mixed collector configuration are here compared to results of the SHRIMP method.

The analyzed in situ zircons are from igneous rock samples formed at $2.2 \mathrm{Ga}, 2.1 \mathrm{Ga}, 0.59,0.41$ and $0.24 \mathrm{Ga}$, and the data obtained by both methods are in agreement when the analytical errors are taken into account. The same spot sites or zircon phases were analyzed for both methods, which is a strong argument for the age comparison between both methods.

The spot sizes for both methods are almost the same, but the calculated individual age errors for each spot are 2 to 4 times greater for the LAMC-ICPMS method when compared to those of the SHRIMP method. The SHRIMP method is therefore a more accurate and precise method, especially due to the flatter spots $(\sim 3 \mu \mathrm{m}=$ SHRIMP and $\sim 15=\mu \mathrm{m}$ for LA-MC-ICPMS) and higher resolution.

The use of masses 202, 204, and 207 in the MIC channels, and 206, 208, and 238 in the Faraday cups are the best configuration for $\mathrm{U}-\mathrm{Pb}$ zircon dating of samples formed in the Phanerozoic or with small amounts of radiogenic ${ }^{207} \mathrm{~Pb}$. One of the main problems of U-Pb zircon dating by LA-ICPMS methods is the common $\mathrm{Pb}$ correction, so it is recommended that those cycles with $206 \mathrm{~Pb} / \mathrm{Pb} 204$ ratios $<1,000$ would be excluded to reduce data.

In this study, the GJ-1 also proved to be a suitable zircon standard for the laser ablation studies.

The advances of LA-MC-ICPMS will be mainly associated with the improvement of the Laser Microprobes (e.g., Fenton Laser), as well as the improvement of MIC channels or similar devices for acquiring small amounts of isotopes with a better precision.

\section{ACKNOWLEDGMENTS}

This work is part of Conselho Nacional de Desenvolvimento Científico e Tecnológico (CNPq) Project \# 310593/2006-9 to FCJ. We thank Léo A. Hartmann and João O. Santos for providing the zircon sample of the Tandilla region to be analyzed by LA-ICPMS. The technicians Fabrício Vieira dos Santos and Maurício Dias Souza are thanked for helping in the sample preparation. We also thank the PETROBRAS and Rede GeoChronos for the financial support for the acquisition of the MC-ICPMS and Laser Microprobe used at the Rio Grande do Sul University.

\section{RESUMO}

O método LA-MC-ICP-MS utilizado para datação U-Pb in situ está cada vez mais preciso e reprodutível devido à melhor desempenho técnico das microssondas a LASER e os espectrômetro de massa com plasma acoplado indutivamente (ICP-MS). Com intuito de testar a validade e a reprodutibilidade deste método, 5 amostras diferentes foram selecionadas (incluindo o padrão Temora 2), com idades entre 2.2 Ga e $246 \mathrm{Ma}$, para serem analisadas por ambos os métodos LA-MC-ICPMS e SHRIMP. Os zircões selecionados foram primeiramente datados com SHRIMP e após foram polidos para que fossem realizadas, na maior parte dos casos, as datações com microssonda a LASER de $213 \mathrm{~nm}$ acoplada a um 
espectrômetro de massa com multicoletor misto (copo Faraday e MIC's), no mesmo local ou fase do grão. Os dados foram obtidos pela ação do laser com tamanho de feixe igual a $25 \mu \mathrm{m}$, e mais raramente de 15 e $40 \mu \mathrm{m}$. A calibração cuidadosa entre o copo Faraday e MIC's foi realizada com solução diluída de $\mathrm{U}-\mathrm{Th}-\mathrm{Pb}$ para calcular a taxa de conversão entre os coletores e o uso de um padrão de comportamento homogêneo e constante durante as análises, o padrão GJ-1, para a calibração externa foram decisivas para a obtenção de resultados confiáveis. Todas as idades obtidas foram concordantes dentro dos erros experimentais. Os erros obtidos pelo método LA-MCICP-MS foram, na maior parte, mais altos do que aqueles obtidos pelo método SHRIMP. A utilização do LA-MCICPMS pode ser vantajosa para obtenção de idades U-Pb in situ, desde que não seja necessária a definição estratigráfica de alta resolução.

Palavras-chave: MC-ICP-MS, SHRIMP, Copos Faraday, Sistema de Contagem de Multiplicadores de ions, Datação in situ de U-Pb em zircão.

\section{REFERENCES}

Ávila JN, Chemale Jr F, Mallmann G, Kawashita K and ARMSTRONG RA. 2006. Combined stratigraphic and isotopic studies of Triassic strata, Cuyo Basin. Argentine Precordillera. Bull Geol Soc Am 118:1088-1098.

BABINSKI M, CHEMALE JR F, VAN SCHMUS WR, HARTMANN LA AND DA Silva LC. 1997. U-Pb and Sm-Nd Geochronology of the Neoproterozoic Granitic-Gneissic Dom Feliciano Belt, Southern Brazil. J S Am Earth Sci 10(3-4): 263-274.

Barredo S, Chemale Jr F, Ávila JN, Marsicano C, Ottone EG AND RAMOS V. 2012. Tectono-sequence stratigraphy and $\mathrm{U}-\mathrm{Pb}$ zircon ages of the Rincon Blanco depocenter, northern, Argentina. Gondwana Res 21(2-3): 624-636.

BLACK LP ET AL. 2004. Improved 206Pb/238U microprobe geochronology by the monitoring of a trace-elementrelated matrix effect; SHRIMP, ID-TIMS,ELA-ICP-MS and oxygen isotope documentation for a series of zircon standards. Chem Geol 205: 115-140.

BOWring SA, ERWIN DH, JIN YG, MARTIN MW, DAVIDEK K AND WANG W. 1998. U/Pb Zircon Geochronology and tempo of the End-Permian Mass Extinction. Science 280: 1039-1045.
Buhn B, Pimentel MM, Matteini M and Dantas EL. 2009. High spatial resolution analysis of $\mathrm{Pb}$ and $\mathrm{U}$ isotopes for geochronology by laser ablation multi-collector inductively coupled plasma spectrometry (LA-MC-ICPMS). An Acad Bras Cienc 81: 99-114.

COCHERIE A AND RoBerT M. 2008. Laser ablation coupled with ICP-MS applied to U-Pb zircon geochronology: A review of recent advances. Gondwana Res 14(4): 597-608.

Compston W, Williams IS And Meyer C. 1984. U-Pb geochronology of zircons from lunar breccia 73217 using a sensitive high-resolution ion-microprobe. J Geophys Res B 98: 525-534.

HARTMANN LA, SANTOS JOS, CingOlani CA AND MCNAUGHTON NJ. 2002.Two Paleoproterozoic Orogenies in the Evolution of the Tandilia Belt, Buenos Aires, as Evidenced by Zircon U-Pb SHRIMP Geochronology. Int Geol Rev 44(6): 528-543.

JACKSON SE, PEARSON NJ, GRIFFIN WL AND BELOUSOVA EA. 2004. The application of laser ablation-inductively coupled plasma-mass spectrometry to in situ $\mathrm{U}-\mathrm{Pb}$ zircon geochronology. Chem Geol 211: 47-69.

Koesler J, Fonneland H, Sylvester P, Tubrett M And PEDERSEN RB. 2002. U-Pb dating of detrital zircons for sediment provenance studies - a comparison of laser ablation ICPMS and SIMS technique. Chem Geol 182: 605-618.

Koesler J AND Sylvester P. 2003. Present trends and the Future of Zircon in Geochronology: Laser Ablation ICPMS. Rev Mineral Geochem 53: 243-275.

LUDWIG KR. 2003. User's Manual for Isoplot/Ex version 3.00 A Geochronology Toolkit for Microsoft Excel, No. 4. Berkeley Geochronological Center, Special Publication, 70 p.

Mundil R, Ludwig KR, Metcalfe I And RenNe PR. 2004. Age and Timing of the Permian Mass Extinctions: $\mathrm{U} / \mathrm{Pb}$ Dating of Closed-System Zircons. Science 305: 1760-1763.

Mundil R, Metcalfe I, Ludwig KR, Renne PR, Oberli F AND NICOLL RS. 2001. Timing of the Permian-Triassic biotic crisis: implications from new zircon $\mathrm{U} / \mathrm{Pb}$ age data (and their limitations) Earth Planet Sci Lett 187: 131-145.

STACEY JS AND KRAMERS JD. 1975. Approximation of terrestrial lead isotope evolutionby a two-stage model. Earth Planet Sci Lett 26: 207-221.

TERA F AND WASSERBURG GJ. 1972. U-Th-Pb systematics in three Apollo 14 basalts and the problem of initial $\mathrm{Pb}$ in lunar rocks. Earth Planet Sci Lett 14: 281-304.

WILLIAMS IS. 1998. U-Th-Pb geochronology by ion microprobe. In: McKibben MA, Shanks III WC and Rydley WI (Eds), Applications of Microanalytical Techniques to Understanding Mineralizing Processes. Rev Econ Geol 7: 1-35.

YoudEN WJ. 1951. Statiscal methods for chemists. J Roy Stastical, New York, Wiley, $126 \mathrm{p}$. 